\title{
Wavepackets in the velocity field of turbulent jets
}

\author{
André V. G. Cavalieri ${ }^{1 *}$, Daniel Rodríguez ${ }^{2,3 \dagger}$ \\ Peter Jordan $^{1 \ddagger}$, Tim Colonius ${ }^{2 \S}$, Yves Gervais ${ }^{1 \uparrow}$ \\ ${ }^{1}$ Institut Pprime, Poitiers, France \\ ${ }^{2}$ California Institute of Technology, Pasadena, CA \\ ${ }^{3}$ Universidad Politécnica de Madrid, Spain
}

\begin{abstract}
We study the velocity field of unforced, high Reynolds number, subsonic jets, issuing from round nozzles with turbulent boundary layers. The objective of the study is to discern the presence of instability waves in such flows and to explore their relationship with the radiated sound. The velocity field is measured using a hot-wire anemometer and a stereoscopic, time-resolved, PIV system, the latter being setup so as to measure three components of velocity in cross-stream planes; the field can thereby be decomposed into frequency and azimuthal Fourier modes. The low-angle sound radiation is measured, synchronously with the PIV acquisition, using a microphone ring array at polar angle, $\theta=20^{\circ}$ (measured with respect to the downstream jet axis).

Consistent with previous observations, the azimuthal wavenumber spectra of the velocity and acoustic pressure fields are quite different. The velocity spectrum exhibits a peak at higher azimuthal wavenumber and the peak is found to scale with the local momentum thickness of the mixing layer. The acoustic pressure field is, on the other hand, predominantly axisymmetric, suggesting an increased relative acoustic efficiency of the axisymmetric mode of the velocity field, a characteristic that can be shown, theoretically, to be due to the radial compactness of the flow. This is confirmed by significant correlations, around $10 \%$, between the axisymmetric modes of the velocity and acoustic pressure fields, these values being significantly higher than those previously reported for two-point flow-acoustic correlations in subsonic jets. The axisymmetric and first helical modes of the velocity field are then compared with solutions of linear Parabolised Stability Equations (PSE) (where the experimental mean velocity field is used as the base flow) to ascertain if these modes correspond to linear instability waves. For all but the lowest frequencies close agreement is obtained for the spatial amplification, up to the end of the potential core. The radial shapes of the linear PSE results also agree with the experimental results over the same region. The results suggests that, despite the broadband character of the turbulence of these unforced jets, the evolution of a certain range of frequencies and azimuthal modes can be modelled as linear instabilities of the mean velocity profile, and that these instabilities are associated with the sound radiated at low polar angles.
\end{abstract}

\section{Introduction}

Since the first observations of coherent structures in turbulent jets, ${ }^{1,2}$ instability waves, or wavepackets, have been postulated as a possible sound source mechanism. ${ }^{1,3-6}$ Wavepackets comprise hydrodynamic waves with amplitude growth, saturation and decay. Their spatial extension is much greater than that of the corresponding turbulence lengthscales, and, as we will show, their fluctuation energy considerably less than that of the eddies which dominate the turbulent kinetic energy. Because of their large spatial extension, wavepackets constitute a non-compact sound source, and, on account of their azimuthal coherence, are more

\footnotetext{
*PhD student, Institut Pprime, 43 rue de l'Aérodrome, 86036 Poitiers, France.

${ }^{\dagger}$ Marie Curie COFUND fellow, California Institute of Technology.

¥Research Scientist, Institut Pprime, 43 rue de l'Aérodrome, 86036 Poitiers, France.

$\S$ Professor, California Institute of Technology.

๑ Professor, Institut Pprime, 40 avenue du Recteur Pineau, 86022 Poitiers, France.
} 
acoustically efficient than the more energetic, but compact, stochastic eddies thought to dominate sound radiation at the time of Lighthill. ${ }^{7}$

Many early studies seeking to compare wavepackets in turbulent jets with stability theory involved forced jets, ${ }^{8,9}$ whose phase-locked disturbances are more easily measured experimentally. But the extension of the conclusions of such studies to their unforced counterparts is not straightforward. In natural jets the signature of azimuthally-coherent wavepackets is clearest in the near pressure field, whose structure is considerably simpler than that of the turbulent velocity field: the hydrodynamic pressure field of a turbulent jet has its fluctuation energy concentrated in a few low-order azimuthal modes, in contrast to the velocity field. ${ }^{10,11}$ The same is true in the irrotational, hydrodynamic, region surrounding the jet, ${ }^{12}$ and measurements using line arrays of microphones in this region reveal a hydrodynamic wave extending several jet diameters downstream of the nozzle exit. ${ }^{13,14}$

Recent results ${ }^{15,16}$ show that this region can indeed be modelled quite accurately as a superposition of linear instability waves with different frequencies and azimuthal modes. It is thus tempting to affirm that such waves are also present in the turbulent velocity field; but this remains to be established. The hydrodynamic waves have small amplitudes, no fixed phase reference, and their signature is therefore difficult to educe from the velocity field of unforced jets (a recent attempt has been made by Kerhervé et al. ${ }^{17}$ using data from a Large Eddy Simulation). The signature is, on the other hand, relatively clear in forced jets ${ }^{1,8,9,18}$ and transitional flows, i.e. jets with laminar boundary layers at the nozzle exit. ${ }^{19-22}$ In both cases the velocity field is dominated by coherent structures, at least for small distances from the nozzle exit. This is clearly not the case for natural jets with turbulent boundary layers at the nozzle exit.

Gudmundsson and Colonius ${ }^{16}$ present a comparison between results of linear Parabolised Stability Equations (hereafter PSE) and the velocity field of unforced jets, measured using PIV in cross sections. In that work the PIV measurements were not time resolved, and so comparison for individual frequencies was not possible; the instability waves had to be superposed prior to comparison with experiment. The results show, nonetheless, an encouraging agreement, and this motivated the present experiment, whose goal is frequency-dependent comparisons between instability wave models and measurements.

We present experimental results that probe the velocity fields of unforced, turbulent jets so as to determine if the low-order azimuthal modes of the velocity field can be modelled as linear instability waves. Linear PSE is used, as in the work of Gudmundsson and Colonius, ${ }^{16}$ as this allows the evolution of wavepackets on a slowly-diverging mean flow to be modelled. Linear PSE is used here as a reference model for hydrodynamic waves resulting from the Kelvin-Helmholtz instability in the initial region of a jet. And while the results constitute a partial validation of linear PSE as an appropriate model, our view is that they provide a more general assessment of the presence of such linear waves in unforced turbulent jets, and of the possibility of using a linear model for turbulent velocity fluctuations if the experimental mean velocity profile is used as the base flow for linearisation.

A further motivation for this study is the observation, by Cavalieri et al. ${ }^{23}$ that the sound field radiated by the jets we study are consistent with sound radiation by non-compact wavepackets with low azimuthal wavenumber. Most notably, the sound field at low polar angles is predominantly axisymmetric, and superdirective, as one would expect from a non-compact, wave-packet, sound source. This suggests that an axisymmetric wavepacket within the turbulent field drives the low-angle radiation, and we investigate this by studying the correlation between the individual azimuthal Fourier modes of the velocity and acoustic fields.

The paper is organised as follows. The experimental setup is described in section II. In section III.A we evaluate the dominant azimuthal wavenumbers in both the velocity and the acoustic fields, and the relationship between the azimuthal modes of turbulence and sound is investigated by means of correlations in section III.B.

We evaluate if the axisymmetric and first helical modes of the velocity field can be modelled as linear instability waves, calculated using linear PSE. A brief description of the computational approach is presented in section IV.A. We perform comprehensive comparisons of this model with the experimental results. We first take advantage of the symmetry properties of circular jets, assessing the axisymmetric mode on the jet centerline, using hot-wire measurements, in section IV.B. To obtain results for the helical modes, and at radial positions other than the centerline, stereoscopic time-resolved PIV measurements are made in cross-stream planes at several axial stations. The comparison of the experimental results with the linear instability results are presented in section IV.C, accompanied by a general discussion regarding instability waves in turbulent jets. 


\section{Experimental setup}

The experiments were performed in the 'Bruit et Vent' anechoic facility at the Centre d'Etudes Aérodynamiques et Thermiques (CEAT), Institut Pprime, Poitiers, France. We have carried out velocity measurements of subsonic jets with acoustic Mach numbers equal to $0.4,0.5$ and 0.6 . The nozzle diameter, $D$, was $0.05 \mathrm{~m}$. With these conditions, the Reynolds number, $\rho U D / \mu$, varies from $4.2 \times 10^{5}$ to $5.7 \times 10^{5}$, where $\rho$ and $\mu$ are, respectively, the density and the viscosity at the nozzle exit.

A convergent section was located upstream of the jet exit, with an area contraction of 31 . This was followed by a straight circular section of length $150 \mathrm{~mm}$; a boundary layer trip was used to force transition $135 \mathrm{~mm}$ upstream $(2.7 \mathrm{D})$ of the nozzle exit. Hot-wire results for the boundary layer at the nozzle exit are shown in figure 1, showing a typical turbulent profile; boundary layer thickness $\delta$ and momentum thickness $\delta_{2}$ for the three jet Mach numbers are shown in table 1 . The present jet setup was previously used for acoustic measurements with an azimuthal microphone ring. ${ }^{23}$
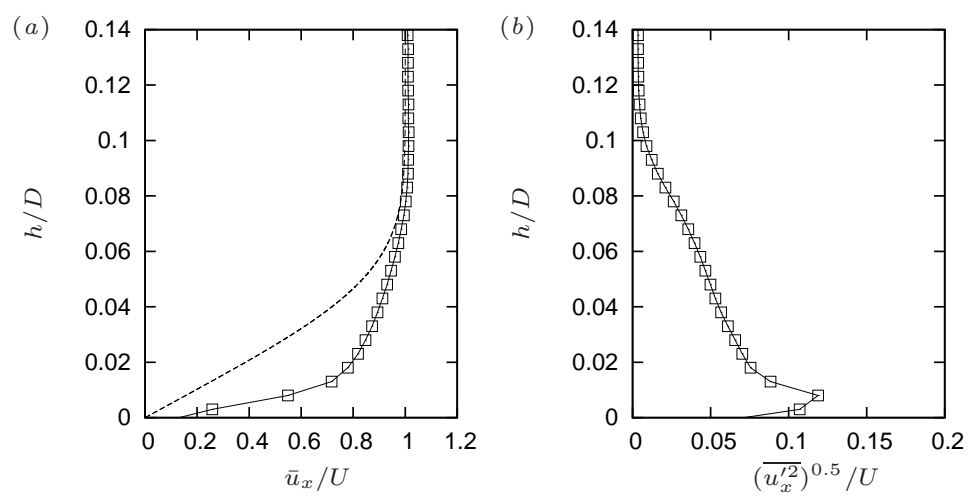

Figure 1. Boundary layer profiles at the nozzle exit for the Mach 0.5 jet: $(a)$ mean velocity and $(b)$ rms value. Dashed line in $(a)$ is Blasius profile.

\begin{tabular}{|ccccc|}
\hline$M$ & $\delta(\mathrm{mm})$ & $\delta / D$ & $\delta_{2}(\mathrm{~mm})$ & $\delta_{2} / D$ \\
\hline 0.4 & 4.5 & $9.0 \cdot 10^{-2}$ & 0.477 & $9.5 \cdot 10^{-3}$ \\
0.5 & 4.25 & $8.5 \cdot 10^{-2}$ & 0.401 & $8.0 \cdot 10^{-3}$ \\
0.6 & 4.25 & $8.5 \cdot 10^{-2}$ & 0.396 & $7.9 \cdot 10^{-3}$ \\
\hline
\end{tabular}

Table 1. Boundary layer thickness $\delta$ and momentum thickness $\delta_{2}$ at the nozzle exit

Velocity measurements were obtained using a traversing single hot wire and with stereoscopic, timeresolved particle image velocimetry (TR-PIV). The hot wire was calibrated in situ using the procedure described by Tutkun et al. ${ }^{24}$ with a traversing Pitot tube giving the mean axial velocity.

The stereoscopic TR-PIV experiments were done with a setup similar to Køenig et al. ${ }^{25}$ The measurements are performed in cross-stream planes, as in Tinney et al. ${ }^{26}$ and Kønig et $a l .{ }^{25}$ Two cameras are placed at an angle of $45^{\circ}$ to the laser plane, one placed upstream and the other downstream of the plane. The flow was seeded with oil smoke. The sampling frequency is $5 \mathrm{kHz}$, which corresponds to St $=1.82,1.46$ and 1.21 for the $M=0.4,0.5$ and 0.6 jets, respectively. A total of 19414 image pairs were recorded. Image-processing consisted of a five-pass correlation routine with $64 \times 64$ pixel correlation for the first pass, $16 \times 16$ pixel for the final pass and with a $50 \%$ correlation overlap at each pass, done with LaVision software Davis 8; this leads to velocity fields with $114 \times 102$ velocity vectors. This grid was subsequently interpolated to cylindrical coordinates to allow the expansion of the velocity field in azimuthal Fourier modes.

We have also measured the acoustic pressure with an azimuthal ring of nine microphones at a polar angle $\theta=20^{\circ}$, with a distance $R$ to the nozzle exit equal to $42.3 \mathrm{D}$. These measurements were done simultaneously with the TR-PIV experiment, which allowed calculation of flow-acoustic correlations in two ways: the first was between a single point in the velocity field and a single microphone, as often done in the litterature; ${ }^{27-31}$ the second was between a given azimuthal mode in velocity and acoustic fields. This last approach has a theoretical motivation, detailed in section III.B, and allows the determination of correlations between instability waves in the flow and the radiated sound field. 
As an evalution of the errors in the present experiment, the TR-PIV results for the axial velocity at $x / D=2$ for the $M=0.4$ jet are compared in figure 2 to the measurements taken with the traversing Pitot tube and hot wire. Close agreement is found for both the mean and rms values of the velocity.
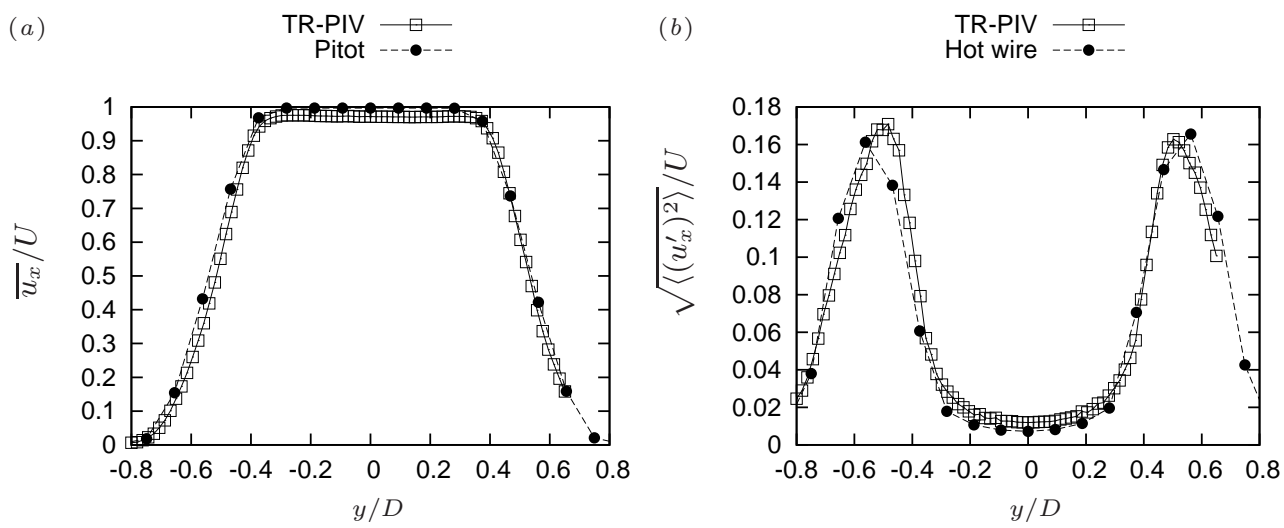

Figure 2. Comparison of (a) mean and (b) rms value of the axial velocity at $x / D=2$ for the $M=0.4$ jet using the different experimental techniques of the present work

The sampling frequency of $5 \mathrm{kHz}$ was the maximum possible value with the available equipment. To evaluate the effect of aliasing in spectra obtained from the TR-PIV measurements, these are compared in figure 3 to hot-wire spectra. For positions close to the jet lipline, such as shown in figures $3(b)$ and $(c)$, the difference between TR-PIV and hot-wire spectra is slight. However, on the jet axis (figure $3 a$ ) the difference becomes significant, particularly for frequencies far from the peak. In addition to the aliasing errors, inside the potential core the amplitudes of velocity fluctuations are much lower than in the regions of turbulent flow, leading to a reduction of the signal-to-noise ratio if one considers that the noise in PIV measurements is uniformly distributed in space. In agreement with this, the errors in the determination of spectra from TR-PIV results are reduced for downstream positions on the jet centerline, as seen in figure $3(d)$.
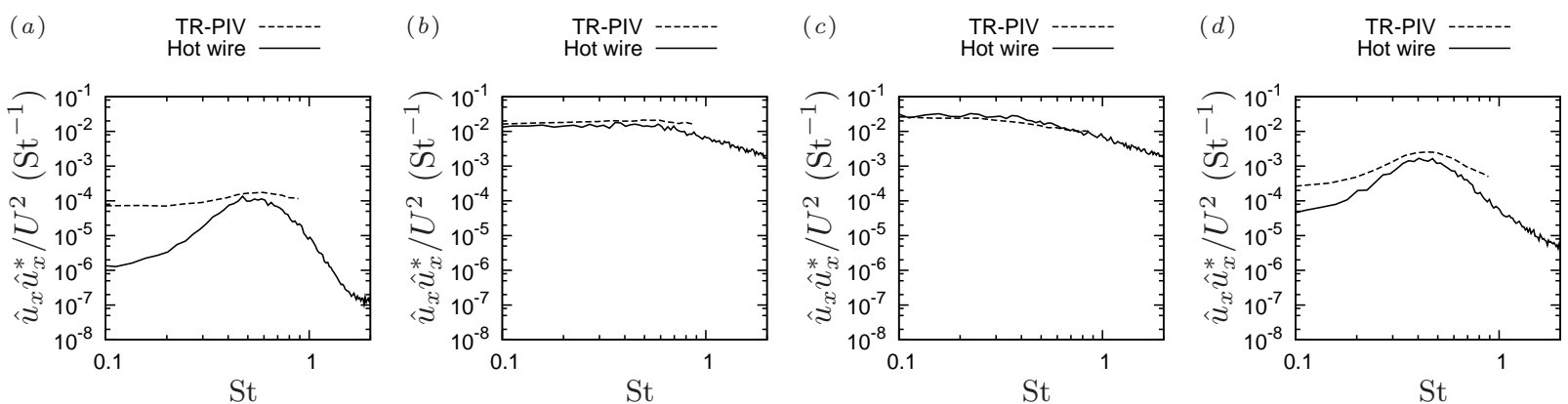

Figure 3. Comparison of hot wire and PIV spectra at $(a) x / D=2, r / D=0,(a) x / D=2, r / D=0.48,(a) x / D=2$, $r / D=0.57$ and $(d) x / D=4, r / D=0$.

To avoid significant errors due to aliasing in the TR-PIV results, we focus the analysis of the $M=0.4$ jet measurements on the Strouhal number range of $0.3 \leq \mathrm{St} \leq 0.7$. For higher Mach numbers, aliasing becomes significant, even for this Strouhal number range; however, its effect is apparently uniform for all radial positions, as shown in Appendix A, where we see also that a correction of the PIV results based on hot-wire measurements leads to close agreement between velocity results for $M=0.6$ and linear PSE solutions.

\section{Azimuthal content of the velocity and acoustic fields}

\section{III.A. Dominant azimuthal wavenumbers for velocity and far-field pressure}

To present a view of the characteristic turbulent structures in the velocity field of the the present jets, we present in figure 4 snapshots of the instantaneous axial velocity fluctuations in a cross section of the Mach 0.4 jet. Azimuthally-coherent structures, such as the ones modelled by linear instability models, are hardly 
visible in this visualisation, which is dominated by smaller-scale eddies with the characteristic turbulent length scale. The simple observation of the fields in figure 4 shows that the present jets are not dominated by coherent structures such as forced jets, ${ }^{1,18}$ and, at first sight, does not suggest the existence of instability waves of low azimuthal wavenumber in such flows.

(a)

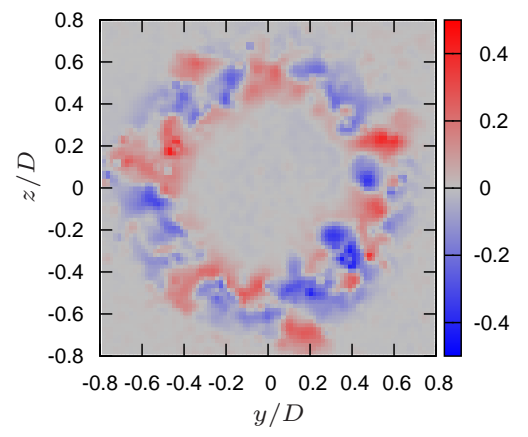

$(b)$

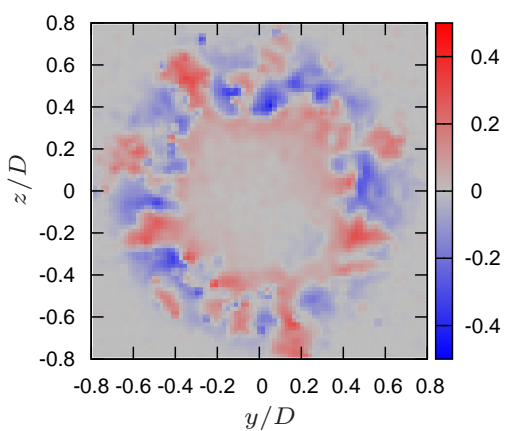

$(c)$

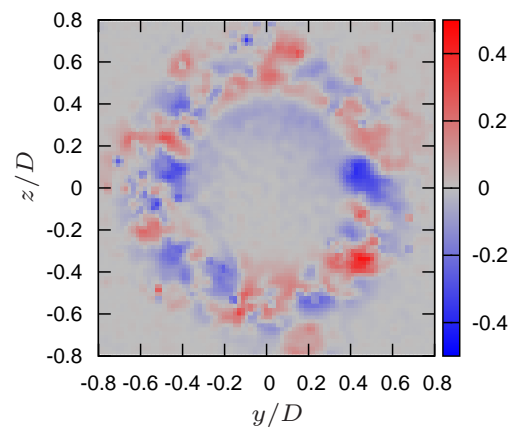

Figure 4. Sample instantaneous experimental axial velocity fluctuations at $x / D=2$ for the Mach 0.4 jet

On the other hand, application of an azimuthal Fourier series to the turbulent field reveals that some energy is contained in lower azimuthal modes, and even for the axisymmetric mode. Figure 5 shows the energy of the fluctuations of axial velocity on the jet lipline, resolved as a function of the azimuthal wavenumber. We notice in figure 5(a) that the most energetic azimuthal mode is around 11 for the near nozzle region $(x / D=1)$. For downstream positions, lower azimuthal modes of velocity become more important, and the peak shifts to $m=4$ for $x / D=3$.

(a)
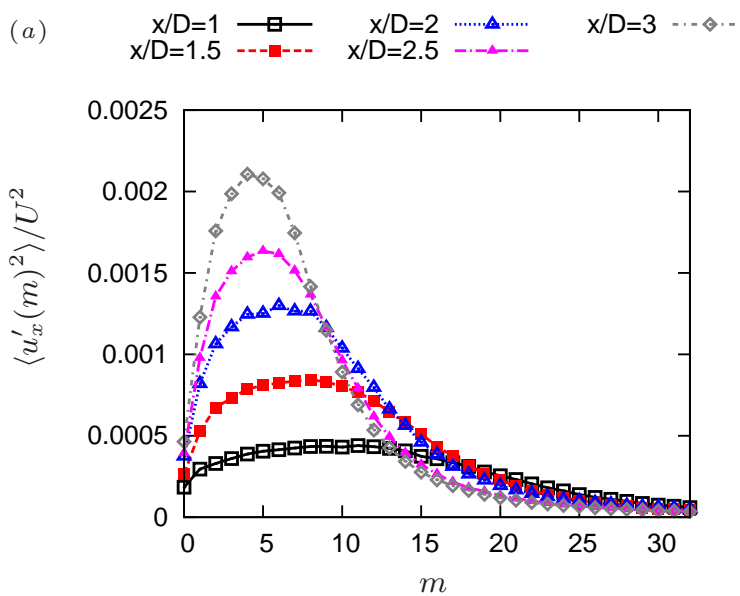
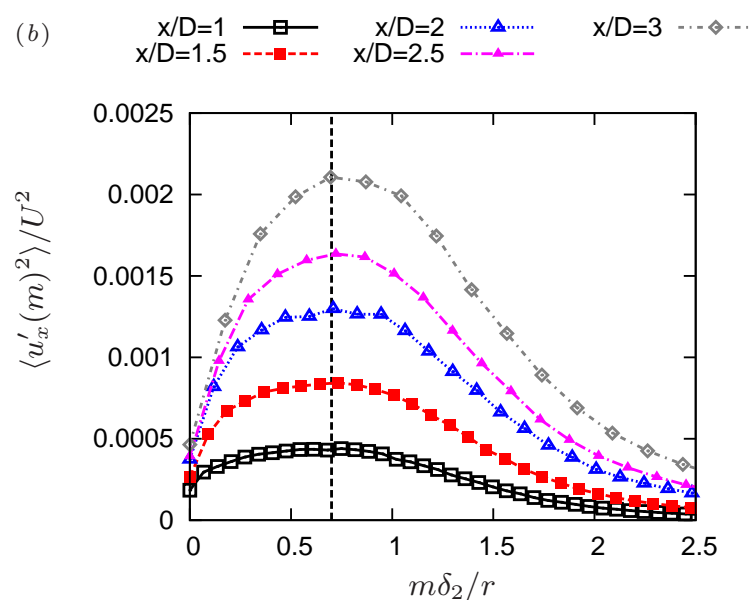

Figure 5. Energy of axial velocity fluctuations on the jet lipline as a function of azimuthal wavenumber scaled with (a) radius $r$ and (b) local momentum thickness $\delta_{2}$. Dashed line in (b) refers to $m \delta_{2} / r=0.7$.

Rescaling of the azimuthal wavenumber with the local momentum thickness $\delta_{2}$ for each axial station, as in figure $5(\mathrm{~b})$, shows that the peak azimuthal wavenumber scales with $\delta_{2}$, similar to what happens in turbulent boundary layers (the results of Tomkins and Adrian ${ }^{32}$ show a peak for spanwise wavenumber of $\left.k_{z} \delta_{2} \approx 1\right)$.

Presented in this way, the velocity field pictured in figure 4 is formed by a number of turbulent eddies. The large-scale, energy-containing structures have azimuthal coherence related to the local momentum thickness. However, we note in figure 5 that the energy for the lower azimuthal modes (e.g. $m=0$ or 1 ) is not zero, despite such modes not being readily visible in an instantaneous view of the jet.

In contrast to the energy at high azimuthal wavenumbers in the velocity field, a number of experimental results $^{12,23,33,34}$ show that the radiated sound field at low polar angles is dominated by low azimuthal modes, especially for low frequencies. Figure 6 shows the far-field spectrum at $\theta=20^{\circ}$ resolved into azimuthal modes for the present $M=0.4$ jet (the acoustic field of this jet, and of the other jets studied in the present work, 
is documented in detail by Cavalieri et $\left.a .^{23}\right)$. We see in figure $6(a)$ that for low angles the sound field is predominantly axisymmetric; this dominance of mode 0 is particularly significant for low Strouhal numbers, as exemplified by the sound pressure level for $\mathrm{St}=0.2$ shown in figure $6(b)$.
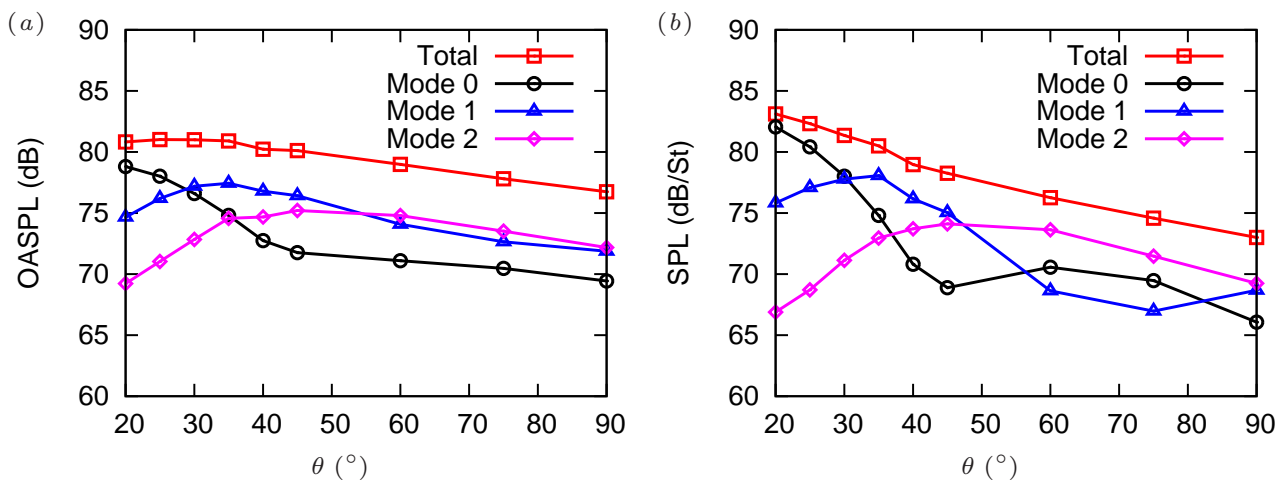

Figure 6. Directivity for the $M=0.4$ jet: $(a)$ overall sound and $(b)$ SPL for St $=0.2$. Taken from Cavalieri $e t$ al. ${ }^{23}$

Theoretically, the difference of the dominant azimuthal wavenumbers in the turbulent and in the acoustic fields can be understood by noting that for low frequencies a condition of radial compactness is satisfied by the jet: the diameter is significantly lower than the acoustic wavelength. In these cases, higher azimuthal modes will have low acoustic efficiency due to the destructive interference in the $\exp (\mathrm{i} m \phi)$ factor in the acoustic source.

Use of cylindrical coordinates and azimuthal modes in Lighthill's analogy allows the quantification of this effect. For low acoustic angles, consideration of azimuthal mode $m$ and frequency $\omega$ of the $T_{x x}$ component of Lighthill's stress tensor leads to

$$
p(R, \theta, m, \omega)=-\frac{\mathrm{i}^{m} k_{a}^{2} \cos ^{2} \theta \mathrm{e}^{-\mathrm{i} k_{a} R}}{2 R} \int \mathrm{e}^{\mathrm{i} k_{a} x \cos \theta} \mathrm{d} x \int T_{x x}(x, r, m, \omega) J_{m}\left(k_{a} r \sin \theta\right) r \mathrm{~d} r,
$$

where $k_{a}$ is the acoustic wavenumber $\omega / c$. The Bessel function of the first kind $J_{m}$ results from azimuthal integration, and leads to a lower efficiency of high azimuthal modes, as explored previously in the litterature. ${ }^{5,10,35,36}$

This effect is illustrated in figure 7. Noting that $k_{a} r=2 \pi \mathrm{St} M r / D$, we see that the ordinate in the figure is the ratio of $J_{m}\left(k_{a} r \sin \theta\right)$ to $J_{0}\left(k_{a} r \sin \theta\right)$ in decibels, and indicates the efficiency of mode $m$ relative to the axisymmetric mode for sound radiation at $\theta=30^{\circ}$. We note that for low Strouhal numbers this acoustic efficiency decays quite fast with increasing azimuthal wavenumber $m$, which suggests that low azimuthal modes in the turbulent field account for most of the sound generation at these low frequencies and polar angles, in spite of their lower energy.

\section{III.B. Correlations between axisymmetric modes of velocity and far-field sound}

Based on the observed contrast between the dominant azimuthal modes in the velocity and far-field pressure, and on the theoretical higher acoustic efficiency of lower azimuthal modes, we have investigated the relationship between azimuthal modes in the jet and in the acoustic field with the computation of correlations between velocity and far-field pressure at $\theta=20^{\circ}$. We present first two-point correlations, defined as

$$
C_{u_{x}, p}(x, r, R, \theta, \tau)=\frac{1}{t_{f}} \int_{0}^{t_{f}} u_{x}(x, r, \phi, t) p(R, \theta, \phi, t+\tau) \mathrm{d} t .
$$

These correlations ignore the difference in the azimuthal content between velocity and pressure fields, and would amount to an attempt to correlate a high azimuthal wavenumber in the velocity field to a low one in the acoustic field.

If we assume instead a relationship between azimuthal modes in the velocity and in the acoustic field, such as in eq. (1), it makes sense to correlate, instead, the axisymmetric modes of velocity and far-field pressure, as

$$
C_{u_{x}, p}^{0}(x, r, R, \theta, \tau)=\frac{1}{t_{f}} \int_{0}^{t_{f}} u_{x}(x, r, m=0, t) p(R, \theta, m=0, t+\tau) \mathrm{d} t,
$$




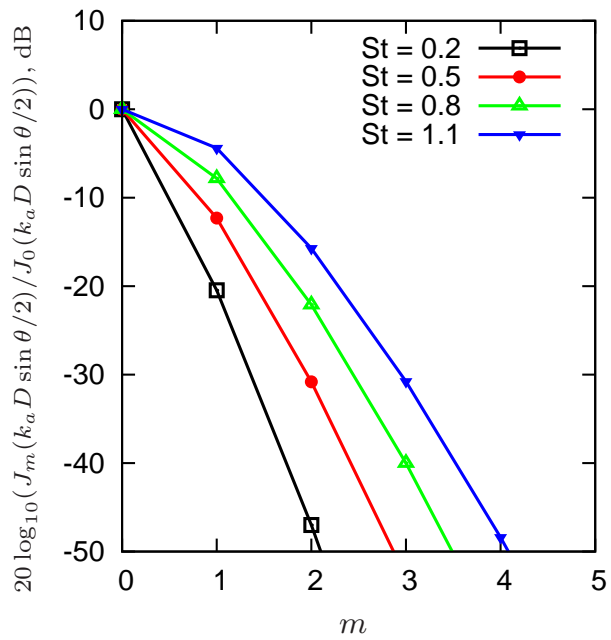

Figure 7. Acoustic efficiency for sound radiation at $\theta=30^{\circ}$ by the azimuthal mode $m$ relative to the axisymmetric mode, for a ring source at $r=D / 2$ in a $M=0.6$ jet.

which isolates the axisymmetric mode of the axial velocity before correlation with the axisymmetric part of the far-field sound.

Results of the two-point correlations, as in eq. (2), are presented in figures 8 and 9 , for the $M=0.4$ and $M=0.6$ jets, respectively. The correlation results have a typical noise level around 0.02 . This is due to the reduced number of TR-PIV samples (19414). We see that the two-point correlations in figures 8 and 9 are low, without an apparent peak above the noise level.
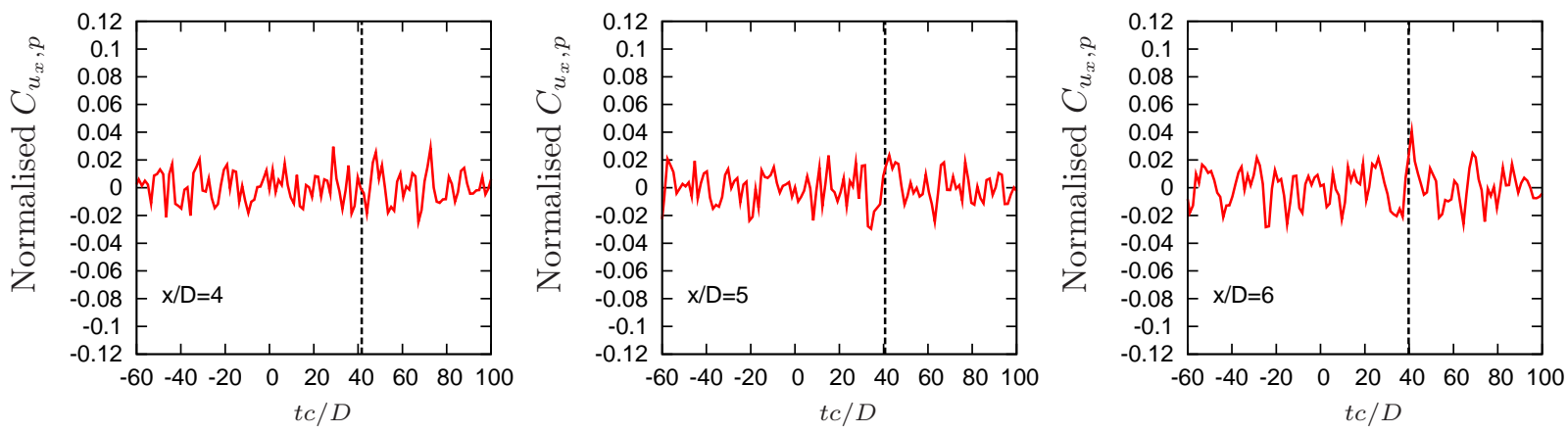

Figure 8. Two-point correlations for the $M=0.4$ jet, taken with a velocity measurement at $r=D / 4$ and far-field pressure at $\theta=20^{\circ}$. The dashed line indicates propagation time without flow-acoustic effects.
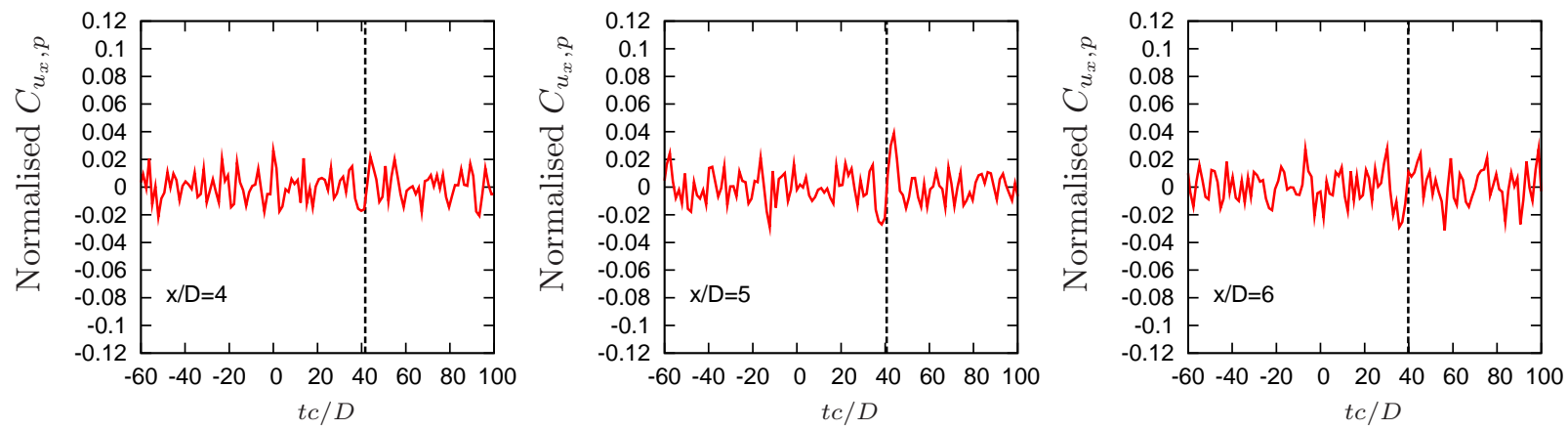

Figure 9. Two-point correlations for the $M=0.6$ jet, taken with a velocity measurement at $r=D / 4$ and far-field pressure at $\theta=20^{\circ}$. The dashed line indicates propagation time without flow-acoustic effects.

Computation of correlations between axisymmetric modes, as in eq. (3), leads to the results shown in figure 10 and 11. The correlations are now well above the noise level, and are of order of $10 \%$ for the 
$M=0.6$ jet, which is significantly higher than values obtained by two-point correlations in previous studies of subsonic turbulent jets. ${ }^{28,30}$
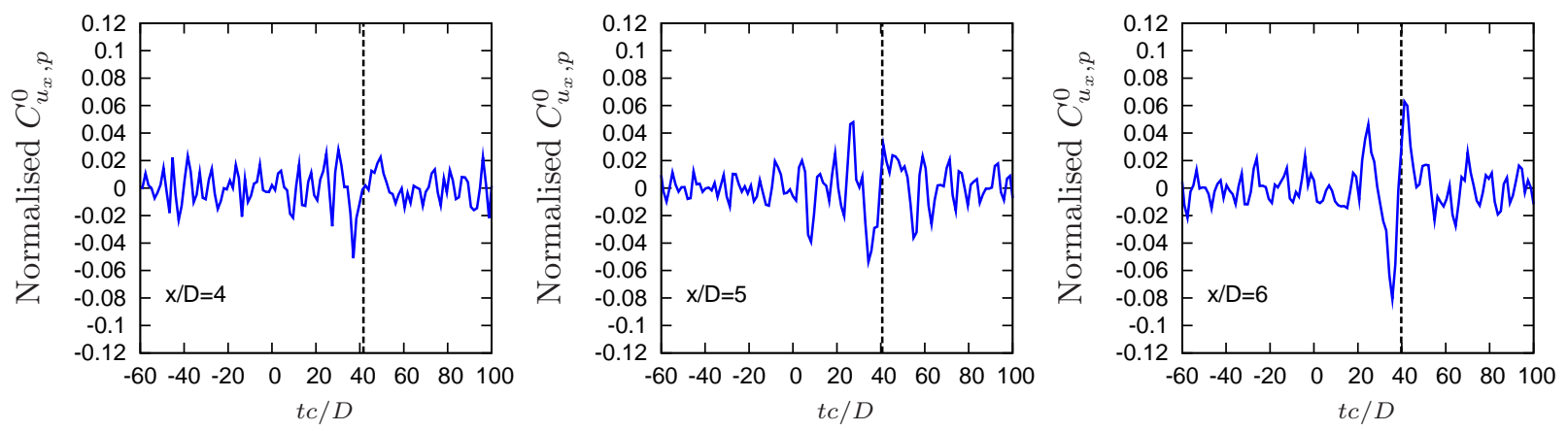

Figure 10. Correlations between mode-0 axial velocity at $r=D / 4$ and mode- 0 far-field pressure at $\theta=20^{\circ}$ for the $M=0.4$ jet. The dashed line indicates average propagation time without flow-acoustic effects.
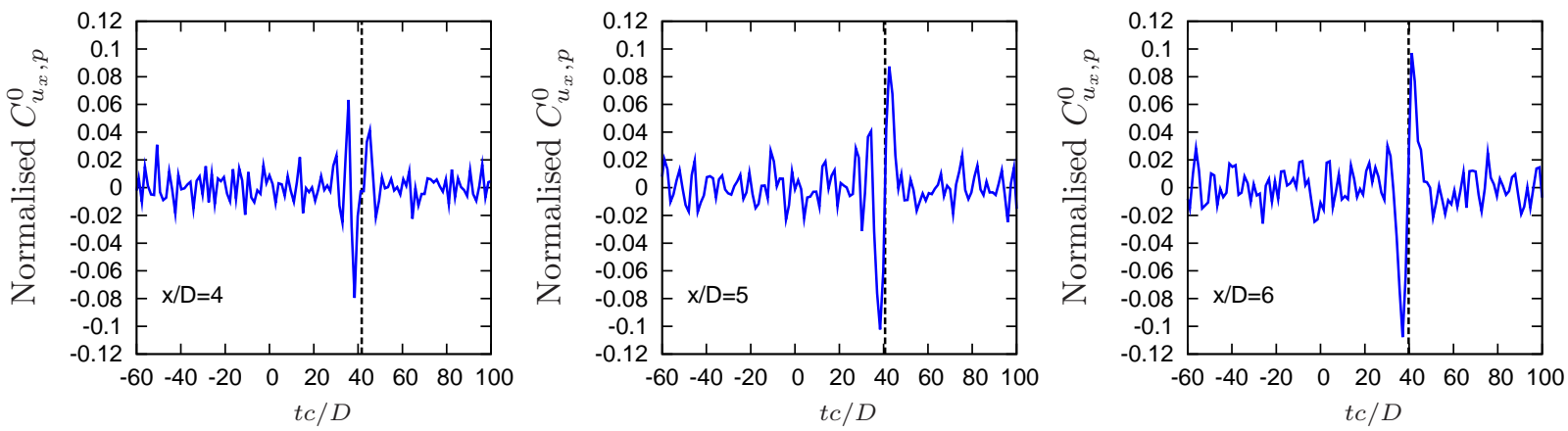

Figure 11. Correlations between mode-0 axial velocity at $r=D / 4$ and mode-0 far-field pressure at $\theta=20^{\circ}$ for the $M=0.6$ jet. The dashed line indicates average propagation time without flow-acoustic effects.

In addition, previous studies showed a strong decay of two-point correlations when the reference position is moved away from the jet centerline; an example is the work of Panda et al. ${ }^{30}$ In Panda et al.'s experiment, all correlations of subsonic jets taken at $r / D=0.45$ were below the experimental noise level. Here we still see significant correlations for several radial positions, as illustrated in figure 12. These results support the idea that the azimuthally-coherent part of the velocity field, which also happens to be radially-coherent, has higher acoustic efficiency, and, despite being less energetic than the dominant azimuthal wavenumbers, is the dominant source of low-angle sound radiation.

Noting that most of the acoustic field at $\theta=20^{\circ}$ is axisymmetric, ${ }^{23}$ the present results show the significance of the axisymmetric velocity mode for sound radiation at low polar angles, and confirm the theoretical predictions. However, care should be taken in the interpretation of these correlation results, since we see that correlations are significant over a region which is extended in both $x$ and $r$; even when $C^{0}$ is used to account for individual azimuthal modes, the correlation is performed between a ring in the flow and a second ring in the acoustic field, and thus no information is obtained about the extended axial structure of the noise source.

The observations in the present section motivate the research for an appropriate model for the lower-order azimuthal modes in a jet, which we develop in the next section as linear instability waves.

\section{Detection of linear instability waves in the velocity field}

Previous experimental observations of the present jets ${ }^{23}$ have shown that the measured acoustic radiation is compatible with a wave-like, non-compact source with low azimuthal wavenumber, and the results of the preceding section show further evidence linking the axisymmetric part of the velocity field and the radiated sound.

In the present section we investigate the lower azimuthal modes of the velocity field to ascertain if they can be modelled as instability waves. We use as a reference solution for linear instability waves a computation 


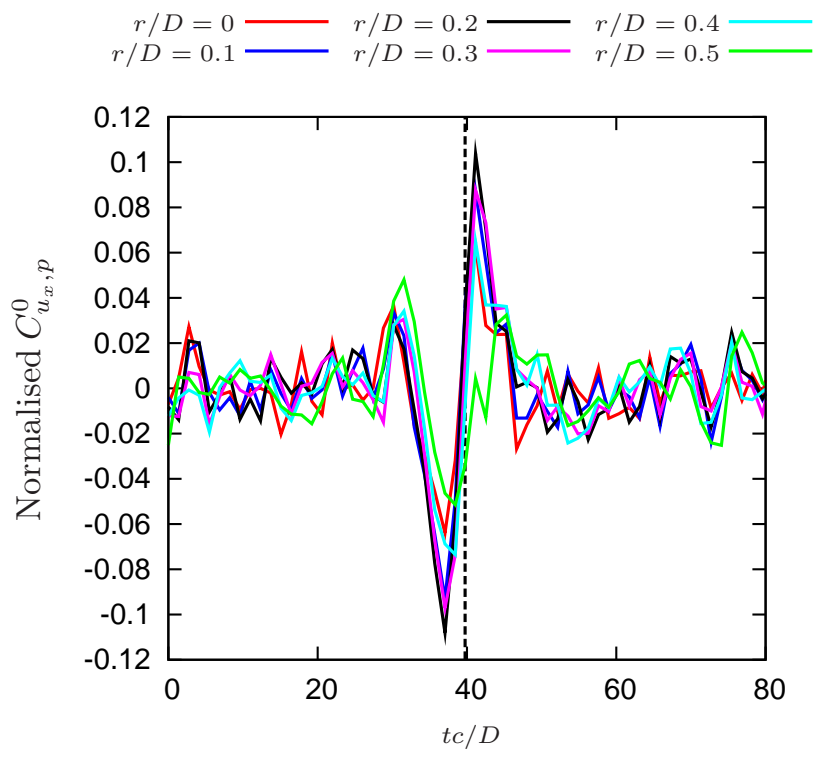

Figure 12. Correlations between mode-0 axial velocity and mode-0 far-field pressure for different radial positions. Results taken at the axial station $x / D=6$ of the $M=0.6$ jet

based on linear Parabolised Stability Equations ${ }^{37}$ using the experimental mean field as a base flow.

Most of the results of the present section refer to the $M=0.4$ jet, since this is the most favourable case for comparison with TR-PIV results due to the aliasing in the experiment. Further comparisons with the $M=0.5$ and $M=0.6$ jets are shown in Appendix A.

\section{IV.A. Linear Parabolised Stability Equations (PSE)}

Instability waves in the jets were modelled using linear PSE, following the approach described by Gudmundsson and Colonius. ${ }^{16}$ We describe the PSE approach briefly in the present section; more details can be found in the cited paper.

PSE represent a generalisation of the parallel-flow linear stability theory for flows with a mild variation on the streamwise direction. For a free jet, the total flow field $q$ is decomposed into a mean (time-averaged) and axisymmetric component $\bar{q}$ and its fluctuations

$$
q=\bar{q}+q^{\prime},
$$

where $q=\left[u_{x}, u_{r}, u_{\phi}, T, \rho\right]^{T}$ is the vector of fluid variables. The fluctuating part is then written as a sum of Fourier modes in the azimuthal direction and in frequency

$$
q^{\prime}(x, r, \theta, t)=\sum_{\omega} \sum_{m=-M}^{M} \chi_{m \omega}(x, r) \exp (\mathrm{i}(m \phi-\omega t)),
$$

where $\chi_{m \omega}$ is the modal function corresponding to the mode $(m, \omega)$.

The mean flow is a function of the axial and radial directions $(x, r)$, but a slow variation of its properties along the axial direction is assumed. This assumption permits the decomposition of $\chi_{m \omega}$ into a slowly varying shape function (that evolves in the same scale as the mean flow) and a rapidly varying wave-like part:

$$
\chi_{m \omega}(x, r)=A_{m \omega}(x) \cdot \hat{q}_{m \omega}(x, r)=\exp \left(\mathrm{i} \int_{x} \alpha_{m \omega}(\xi) d \xi\right) \cdot \hat{q}_{m \omega}(x, r) .
$$

Here $\alpha_{m \omega}(x)$ is a complex axial wavenumber, for which a mild variation is also assumed. It is important to stress that the separation in scales between the mean flow, and the modal shape functions on one hand, and the modal wavelengths associated with $\alpha_{m \omega}$ on the other is a necessary hypothesis in the derivation of the PSE. However, for low frequencies the wavelength can be comparable to the extent of the potential core. 
We introduce the previous decomposition into the compressible Navier-Stokes, continuity and energy equations. After subtraction of the terms corresponding to the mean flow, and neglecting quadratic terms on the fluctuations, we arrive at the system of equations

$$
\begin{gathered}
\left(\mathcal{L}^{0}-\mathrm{i} \omega \mathcal{L}^{t}+\mathcal{L}^{x} \frac{\partial}{\partial x}+\mathcal{L}^{r} \frac{\partial}{\partial r}+\mathrm{i} m \frac{\mathcal{L}^{\theta}}{r}+\right) \hat{q} \\
+\frac{1}{R e_{a}}\left(\mathcal{V}^{0}+\mathcal{V}^{x} \frac{\partial}{\partial x}+\mathcal{V}^{r} \frac{\partial}{\partial r}+\mathrm{i} m \frac{\mathcal{V}^{\theta}}{r}+\mathcal{V}^{x x} \frac{\partial^{2}}{\partial x^{2}}+\mathcal{V}^{r r} \frac{\partial^{2}}{\partial r^{2}}\right. \\
\left.-m^{2} \frac{\mathcal{V}^{\theta \theta}}{r^{2}}+\mathcal{V}^{x r} \frac{\partial^{2}}{\partial x \partial r}+\mathrm{i} m \frac{\mathcal{V}^{r \theta}}{r} \frac{\partial}{\partial r}+\mathrm{i} m \frac{\mathcal{V}^{x \theta}}{r} \frac{\partial}{\partial x}\right) \hat{q}=0
\end{gathered}
$$

The linear operators $\mathcal{L}$ and $\mathcal{V}$ can be found elsewhere. ${ }^{38}$ For brevity, the subscripts have been dropped from the shape function and wavenumber in the previous expression. The left-hand-side on (7) is a linear spatial operator for the mode $(m, \omega)$, and each frequency-azimuthal mode component evolves independently of the others. Following from the slow axial variation assumed for $\hat{q}$, the second axial derivatives on the viscous terms are neglected, so that the system of equations can be integrated along the $x$-direction. The decomposition of (6) is ambiguous in that the evolution of $\chi$ can be absorbed into either the shape function $\hat{q}_{m \omega}$ or the complex amplitude $A_{m \omega}$ corresponding to the wave-like behavior. Following Herbert, ${ }^{37}$ the normalisation condition

$$
\int_{0}^{\infty} \hat{q}^{*} \frac{\partial \hat{q}}{\partial x} r d r=0,
$$

where the superscript $*$ denotes complex conjugation, is imposed individually to every mode, removing the exponential dependence on the shape function $\hat{q}$.

The characteristic boundary conditions of Thompson ${ }^{39}$ are used for the outer boundary, while centerline conditions are derived following Mohseni and Colonius. ${ }^{40}$ The same approach of Gudmundsson and Colonius $^{16}$ was applied to obtain numerical solutions of the system of equations (7). The reader is referred to that paper for further details on the computational method.

Adequate boundary conditions are required at the inlet, especially for $\hat{q}$ and $\alpha$, for each mode. The complex amplitudes $A_{m \omega}$ can be rescaled after the computations in order to fit experimental measurements, as linear PSE results are independent of the modal amplitudes and phases. A 'local' spatial linear instability eigenvalue problem (EVP) can be derived from the equations (7); from its solution, the dominant inflectional instability eigenmode is taken as initial condition for $\hat{q}$ and $\alpha$.

\section{IV.B. Comparison with experimental velocity fluctuations on the jet centerline}

The linear modes have a free amplitude, and this has been adjusted using the velocity spectra on the jet centerline. There the kinematic boundary conditions are zero transverse velocity and finite axial velocity for azimuthal mode 0, zero axial velocity and finite transverse velocity for mode 1, and zero velocities for all higher modes. ${ }^{41}$ As the velocity measurements were performed with a single hot wire, we expect that in the potential core the measurements will be of the axial velocity, allowing thus the comparison between the mode 0 from linear PSE and the hot wire spectra.

Figure 13 shows comparison, between linear PSE and experiment, of the amplitude for the streamwise velocity component for the Mach 0.4 jet. The free constant multiplying linear PSE amplitudes was chosen by matching amplitudes at $x / D=2$. Between the nozzle exit and the end of the potential core $(x / D \approx$ $5-5.5)$ there is an amplification of four orders of magnitude of the fluctuation energy in the experiment. In this region there is close agreement between linear PSE and the experimental values for Strouhal numbers of $0.3-0.9$.

We note in figure 13 differences between the modelled instability waves and the experimental results for points downstream of the end of the potential core. Similar behaviour was also observed in previous work by Suzuki and Colonius ${ }^{15}$ and Gudmundsson and Colonius. ${ }^{16}$ In these papers the discrepancies were attributed to fluctuations that were uncorrelated with the upstream instability waves. Proper Orthogonal Decomposition (POD) was applied to obtain modes correlated axially, and the use of the first POD modes allowed uncorrelated oscillations to be filtered, improving significantly the agreement at downstream positions. However, since the hot-wire measurements are single-point we could not apply a POD to these results to verify if this also appplies also for the current experiments. 

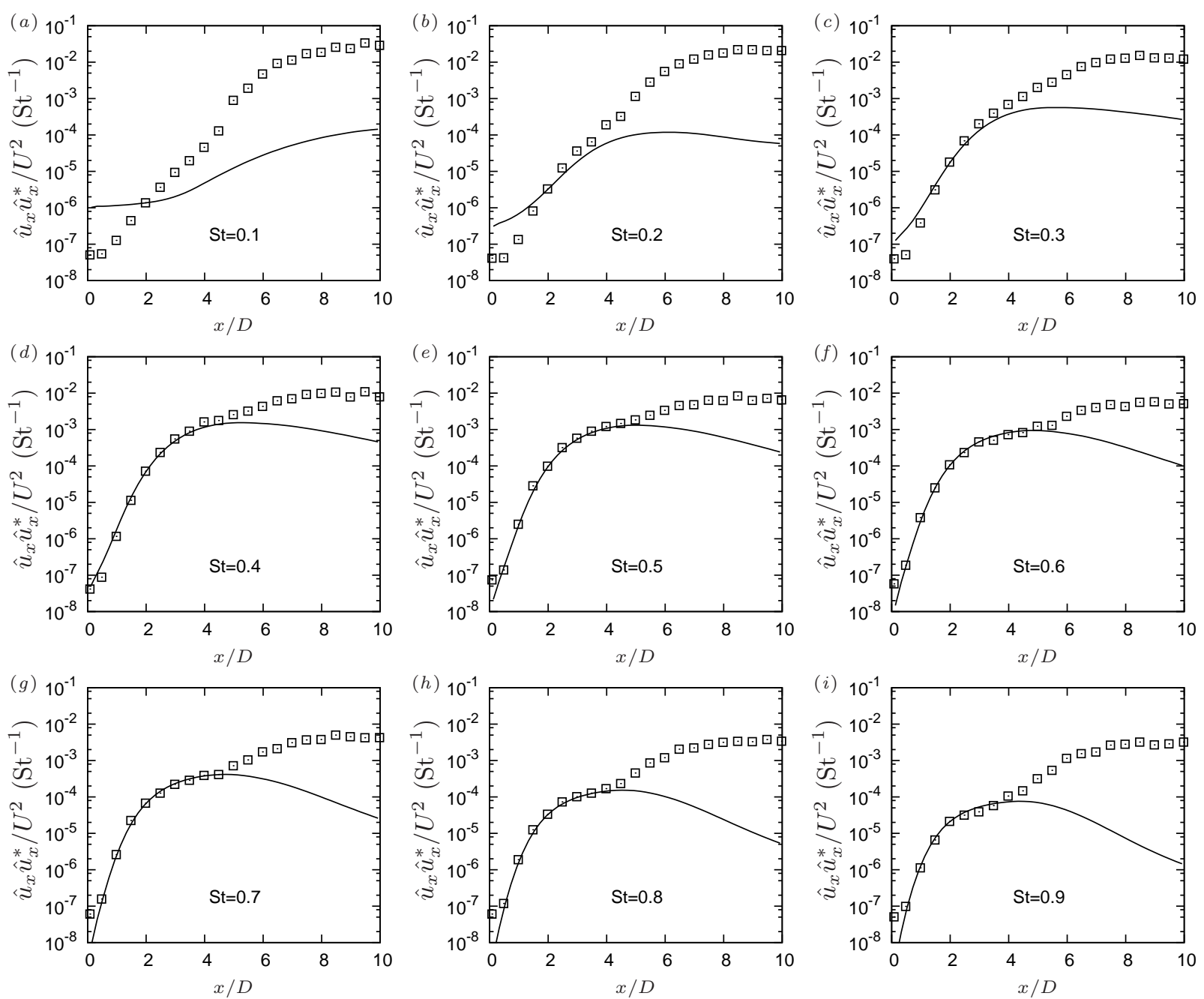

Figure 13. Comparison between linear PSE (lines) for $m=0$ and experimental velocity fluctuations on the centerline (symbols) for the $M=0.4$ jet. Subfigures $(a)-(i)$ refer respectively to Strouhal numbers from 0.1 to 0.9 with increments of 0.1 .

For the two lower Strouhal numbers, 0.1 and 0.2, shown in figures 13( $a$ ) and $(b)$, linear PSE underpredicts the growth rate of the axisymmetric mode. This was also the case in other works ${ }^{15,16}$ and as shown in Appendix A, is also verified for the higher Mach numbers. These discrepancies have been attributed in the cited papers to increased non-parallel effects for these lower frequencies, since the potential core length becomes comparable to the wavelength for these Strouhal numbers, which invalidates the hypotheses in the PSE derivation shown in section IV.A. The lack of agreement at low Strouhal numbers may also be due to non-linear effects; such a possibility has been considered by Sandham and Salgado ${ }^{42}$ and Suponitsky et al., ${ }^{43}$ who show in computations how the non-linear interaction between waves at higher Strouhal numbers may affect the development of low-St velocity fluctuations and change the radiated sound field. The present results do not, however, allow us to discern between these two possibilities to explain the said discrepancies.

Similar agreement is found for the other jets over the same St range; this is shown in Appendix A. However, due to the compressibility effects discussed by Cavalieri et al. ${ }^{23}$ the normalised amplitudes of the linear PSE modes on the jet centerline become lower as the Mach number is increased. This is shown in figure 14 for three Strouhal numbers. We note that the amplitudes near the nozzle exit are quite close, and lower growth rates due to compressibility lead to decreases in amplitudes for higher Mach numbers. The linear PSE modes are seen to correctly model these observed trends in the experimental fluctuations. 

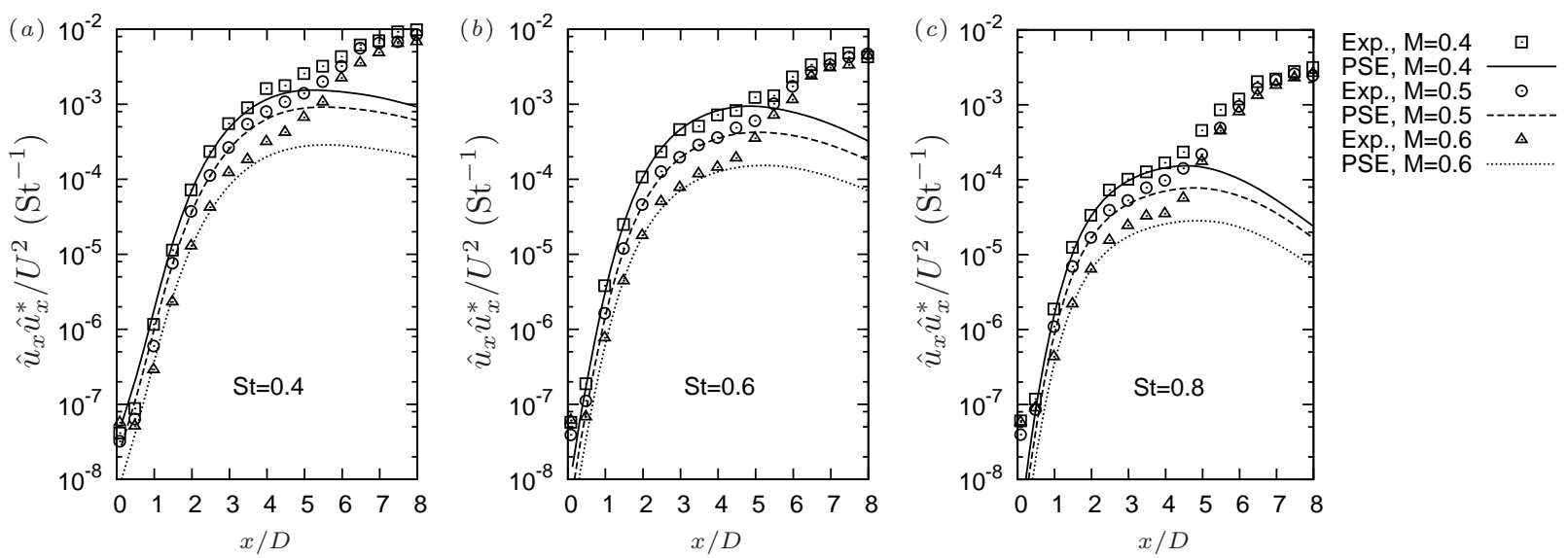

Figure 14. Compressibility effect in the velocity fluctuations and in the linear PSE amplitudes for $(a)$ St $=0.4$, $(b)$ $\mathrm{St}=0.6$ and $(c) \mathrm{St}=0.8$.

\section{IV.C. Cross-stream planes}

Velocity measurements using time-resolved PIV in cross-stream planes of the jet allow application of a Fourier transform in time and of a Fourier series in azimuth, leading to a decomposition of the velocity field in both frequency and azimuthal wavenumber, such as in eq. (5). This allows in turn comparison of experiment with linear PSE results for each $(\omega, m)$ pair at several axial and radial positions.

We show first comparisons for the axisymmetric mode at $x / D=2$ in section IV.C.1 to explain some of the general features of both experiment and PSE solution. The first helical mode is compared to linear PSE at the same axial station in section IV.C.2. Finally, the axial development of instability waves is studied in section IV.C.3. All comparisons in the present section were made for the $M=0.4$ jet.

Throughout this section, comparisons are restricted to the Strouhal number range of $0.3-0.7$. Comparisons for Strouhal numbers of 0.1 and 0.2 , not shown here, did not show good agreement with linear instability wave models, similarly to what was verified for the hot-wire data in section IV.B.

\section{IV.C.1. Axisymmetric mode at $x / D=2$}

The stereoscopic TR-PIV results were compared to the radial shapes of the PSE solutions for azimuthal modes $m=0,1$. We begin the study of linear instability waves by looking at the axisymmetric mode of the $M=0.4$ jet in the present section, focusing first on jet cross section at $x / D=2$. In all comparisons of the axisymmetric mode obtained by TR-PIV results we have used the linear PSE results with amplitudes for $u_{x}$ matched using the hot-wire spectra at $x / D=2$ and $r / D=0$; hence, no further adjustement was performed to match the PIV results. Figure 15 shows a comparison of the axial velocity fluctuations at this station for $m=0$.

As seen in section III.A, the fluctuation energy of the axisymmetric mode close to the jet lipline is much lower than the overall flucutation energy, shown in dash-dot lines in figure 15. This is in contrast to the jet centerline, where all axial velocity fluctuations correspond, as expected, to the axisymmetric mode.

The comparison in figure 15 between PSE and experimental mode 0 shows a fair agreement. However, the PSE solution presents nearly zero amplitudes at a radial position close to the lipline (the precise position depends on the frequency). To understand the meaning of this zero amplitude, we see in figure 16 a comparison of the phase between experiment and linear PSE for Strouhal number of 0.7. We note that in the experiment there is a phase jump of $\pi$ between the two sides of the mixing layer, a behaviour also represented by the PSE solution.

This phase opposition for the streamwise velocity is found in coherent, axisymmetric vortical structures obtained by phase averages of forced jets, ${ }^{8,9,18}$ and was also seen previously in unforced jets. ${ }^{44}$ This is illustrated in figure 16(b). At the radial position of the center of a vortex, shown with a dashed line, the axial velocity is zero; therefore, a forced jet consisting mostly of periodic vortex rings presents a nearly zero amplitude for the streamwise velocity at the radial position of the vortex centers. We can conjecture that the jitter of coherent structures in an unforced jet will lead to temporal changes in the radial position of the centers, so that the experimental amplitude is not zero, as sketched in figure 16(c). 

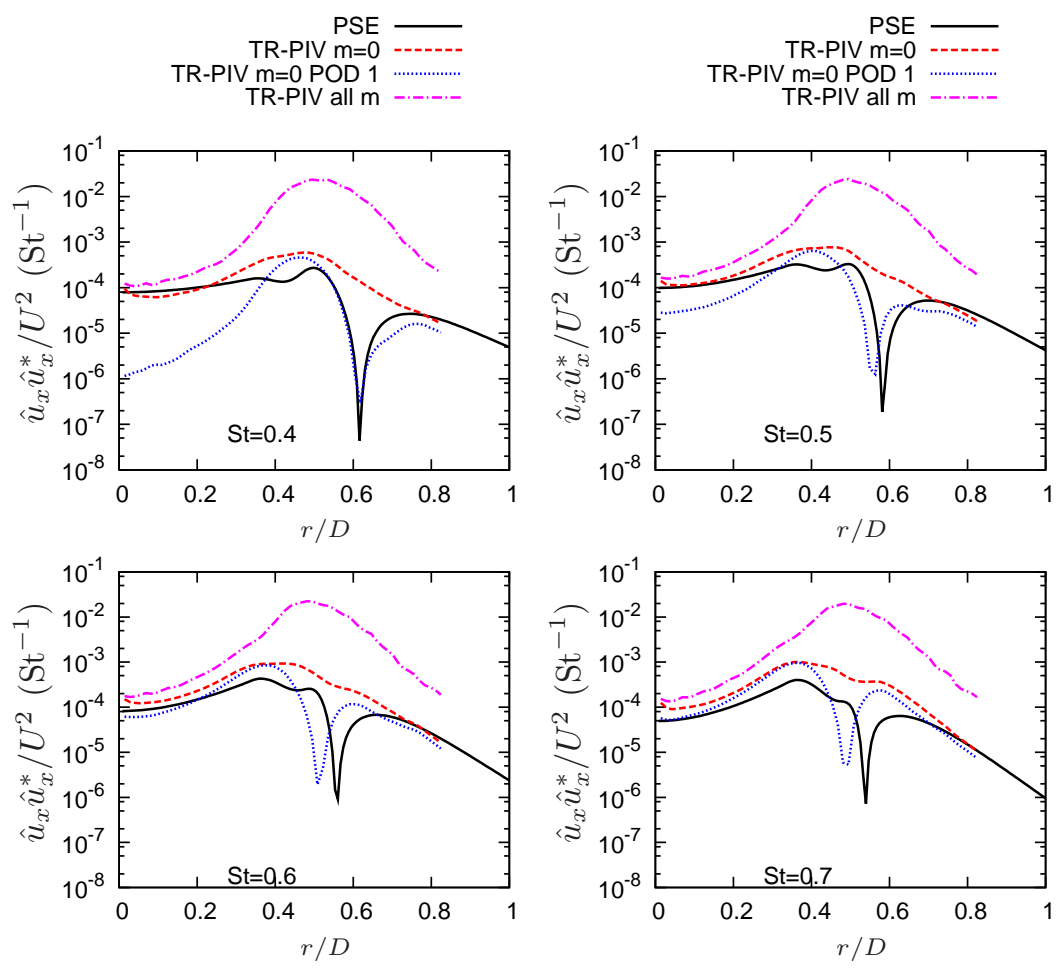

Figure 15. Comparison between $u_{x}$ from linear PSE and experiment for $m=0, M=0.4$ and $x / D=2$

(a)

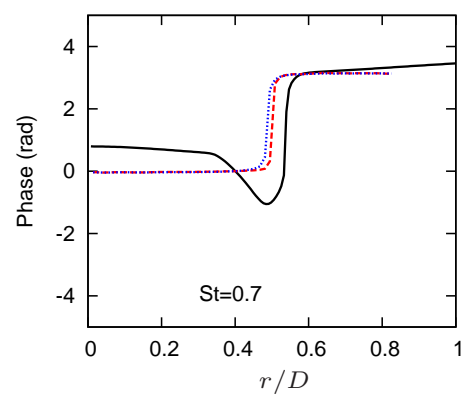

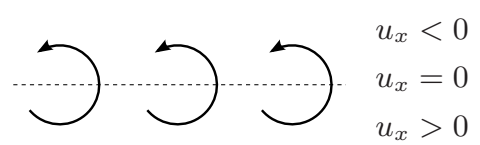

$(c)$

Figure 16. (a) Phases of the streamwise velocity for the axisymmetric mode at $\mathrm{St}=0.7$ and $x / D=2(\mathrm{phase}$ is fixed as zero at $r / D=0.4) ;(b)$ sketch representing the phase difference across a mixing layer with a developping Kelvin-Helmholtz instability; $(c)$ effect of jitter in the position of vortex centers

Based on this conjecture, in an attempt to obtain an average structure of the streamwise velocity without jitter, we have applied spectral POD in the radial direction to extract the correlated part of the fluctuations, using the same approach described by Jung et al., ${ }^{45}$ which amounts to solving the integral equation

$$
\int R_{x, x}\left(x, r, r^{\prime}, m, \omega\right) \xi_{x}\left(x, r^{\prime}, m, \omega\right) r^{\prime} \mathrm{d} r^{\prime}=\lambda(x, m, \omega) \xi_{x}(x, r, m, \omega)
$$

where $\xi$ is an eigenfunction (POD mode), $\lambda$ is the corresponding eigenvalue and and $R_{x, x}$ is given by

$$
R_{x, x}\left(x, r, r^{\prime}, m, \omega\right)=u_{x}(x, r, m, \omega) u_{x}^{*}\left(x, r^{\prime}, m, \omega\right)
$$

This kernel $R_{x, x}\left(x, r, r^{\prime}, m, \omega\right) r$ is not Hermitian; an auxiliar Hermitian kernel was obtained, as in Jung et al., ${ }^{45}$ by multiplying the integral equation (9) by $\sqrt{r}$ and considering $R_{x, x}\left(x, r, r^{\prime}, m, \omega\right) \sqrt{r^{\prime} r}$ as the kernel and $\xi_{x}(x, r, m, \omega) \sqrt{r^{\prime}}$ as the eigenfunction. 
The POD defined in eq. (9) was done for each velocity component independently. We have also applied a POD with the three velocity components in the kernel, as done by Delville, ${ }^{46}$ and results are shown in Appendix B. However, for the presentation of the results we have preferred the scalar POD, since, as shown in Appendix B, the vectorial POD led to nearly identical results, but with the velocity components split into different modes, suggesting that a single physical phenomenon was separated in different POD modes. The scalar POD was considered less ambiguous, and, as will be seen, linear instability waves were consistently detected using the first scalar POD mode for each velocity component.

Throughout this section we have used the projection of the experimental results onto the mode with highest energy, obtained by

$$
\operatorname{POD}\left(u_{x}\right)=\sqrt{\lambda^{(1)}} \xi_{x}^{(1)}
$$

where $\lambda^{(1)}$ is the highest eigenvalue and $\xi_{x}^{(1)}$ the corresponding eigenfunction. This projection was used to extract the coherent part of the velocity field, and is referred to as the "first POD mode" through the remainder of this paper.

This first POD mode for $u_{x}$ is plotted in figures 15 and 16, where we see that this mode presents indeed an amplitude close to zero near the lipline, at the position of the phase jump. The position of this zero amplitude agrees closely with the PSE solution, as shown in figure 15.

The radial velocity is compared in figure 17. Note that linear PSE solutions have the same free constant multiplying all flow variables; therefore, no further adjustement is done for this comparison. The agreement between linear PSE and experiment is good, especially considering the first POD mode close to the centerline. Since the radial velocity should be zero on the centerline for $m=0$, the present results suggest that POD acts in this case as a filter, removing some of the uncorrelated noise in the experimental results and leading to the correct trend for the radial velocity close to the centerline.
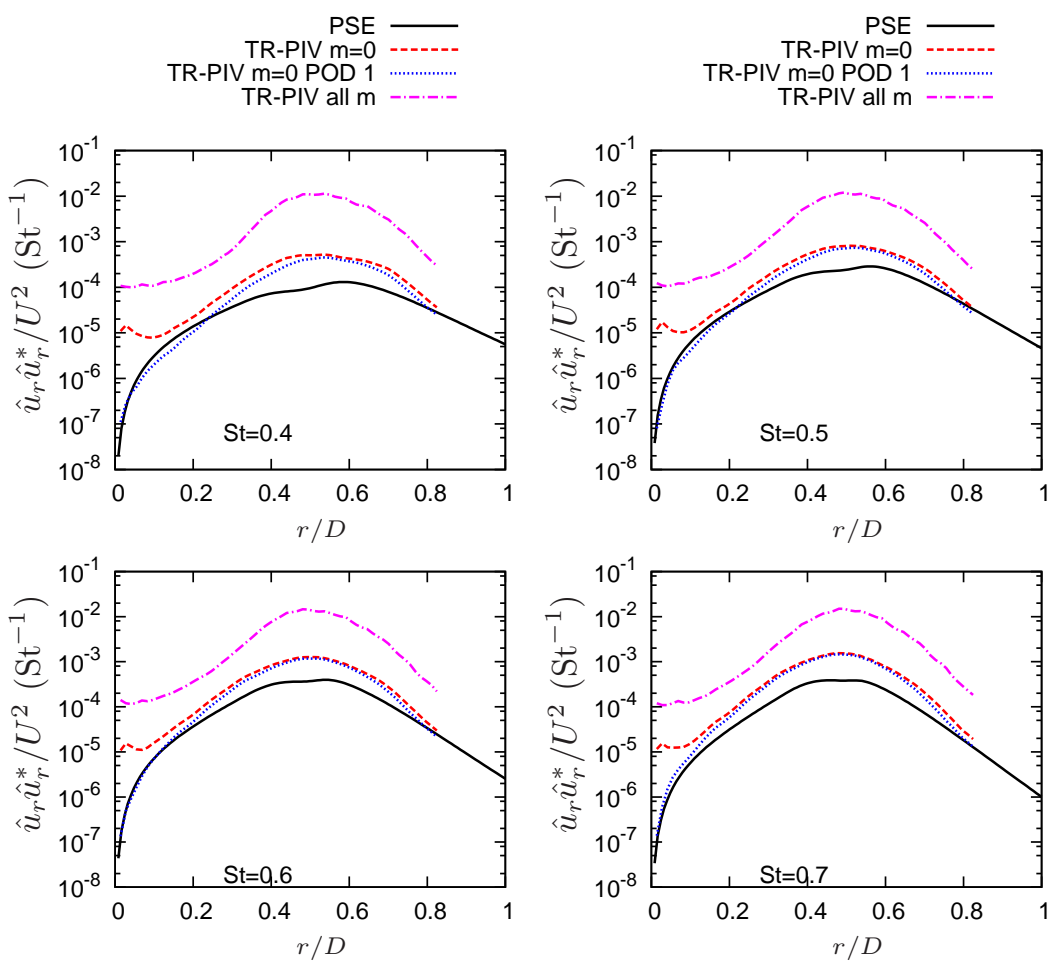

Figure 17. Comparison between $u_{r}$ from linear PSE and experiment for $m=0, M=0.4$ and $x / D=2$

\section{IV.C.2. First helical mode at $x / D=2$}

As in the axisymmetric case, linear PSE solutions have a free constant that is determined using experimental data. However, since the hot-wire measurments of axial velocity on the centerline cannot give information about helical modes, we have used the TR-PIV results at $x / D=2$ to determine this free amplitude for $m=1$. We define an inner product 


$$
\begin{gathered}
\langle u(x, r, \omega, m), \varphi(x, r, \omega, m)\rangle=\int\left[u_{x}(x, r, \omega, m) \varphi_{x}^{*}(x, r, \omega, m)\right. \\
\left.+u_{r}(x, r, \omega, m) \varphi_{r}^{*}(x, r, \omega, m)+u_{\phi}(x, r, \omega, m) \varphi_{\phi}^{*}(x, r, \omega, m)\right] r \mathrm{~d} r
\end{gathered}
$$

between the experimental results and the linear PSE solutions, where $\varphi$ refers to the velocity modelled by the PSE solution $\chi_{m \omega}$. If the velocity field can be expressed as

$$
u(x, r, \omega, m)=b(x, \omega, m) \varphi(x, r, \omega, m),
$$

the value of $b$ can be determined as

$$
b(x, \omega, m)=\frac{\langle u(x, r, \omega, m), \varphi(x, r, \omega, m)\rangle}{\|\varphi(x, r, \omega, m)\|^{2}} .
$$

We have used the results at $x / D=2$ to determine $b(x=2 D, \omega, m)$, and used this value of $b$ as the free constant multiplying the linear PSE results for all values of $x$ and $r$.

Figure 18 presents comparisons for the axial velocity at $x / D=2$. The radial velocity in the same axial station is compared in figure 19. We note that, as for the axisymmetric mode, the solution of linear PSE presents a nearly zero amplitude close to the jet lipline, which compares favourably with the first POD mode calculated from the experimental data. The agreement between model and experiment is good for both the axial and radial velocity components, although for $m=1$ the agreement is not as close as for the axisymmetric mode shown in section IV.C.1.
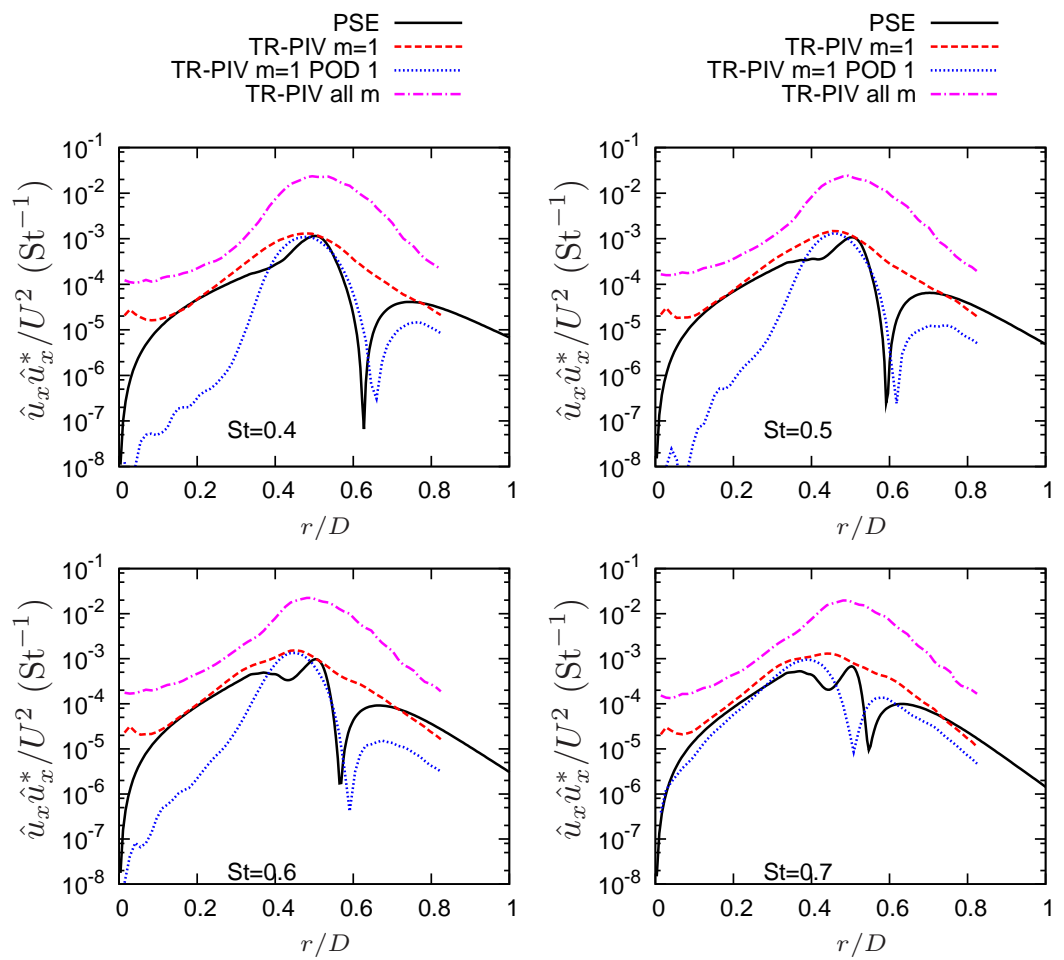

Figure 18. Comparison between $u_{x}$ from linear PSE and experiment for $m=1, M=0.4$ and $x / D=2$

\section{IV.C.3. Axial development of instability waves}

We illustrate the development of linear instability waves in the jet by comparing the linear PSE solution for Strouhal number of 0.5 to the experimental results for both the axisymmetric and first helical modes at axial stations ranging from $x / D=1$ to $x / D=8$. This comparison is shown for the axisymmetric mode in figure 20 for the axial velocity, and in figure 21 for the radial velocity fluctuations. 

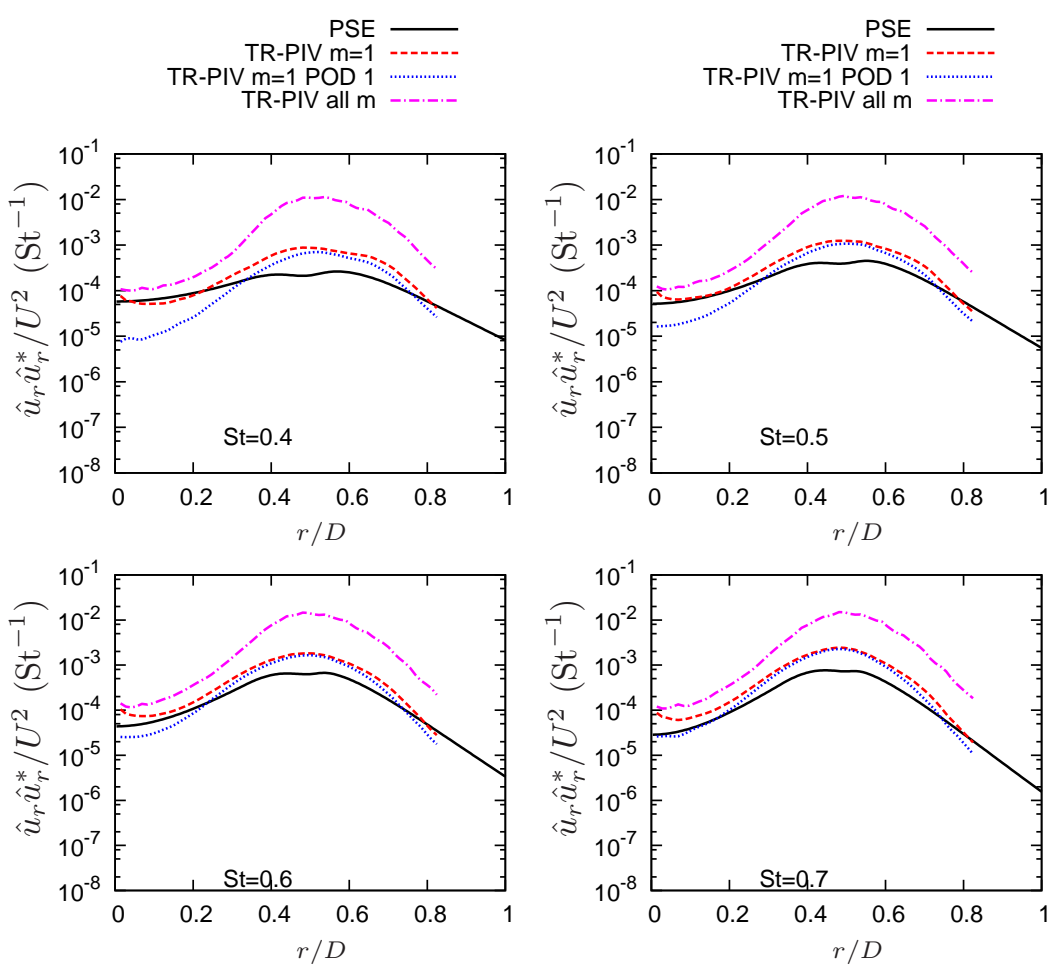

Figure 19. Comparison between $u_{r}$ from linear PSE and experiment for $m=1, M=0.4$ and $x / D=2$

We see in figures 20 and 21 three distinct zones in the jet with regard to the development of instability waves. In a first region, exemplified with the results for $x / D=1$ and $x / D=1.5$, the velocity fluctuations present some differences from the linear instability model, suggesting that this near-nozzle region is characterised by a transition from the fluctuations internal to the nozzle to the Kelvin-Helmholtz instability observed downstream. This transition is more clearly seen in the fluctuations close to the jet lipline; on the centerline, the flow seems to conform earlier to a jet instability, at, say, $x / D=0.5$, as seen in the hot-wire results in section IV.B. Another possibility to explain results for low $x$ is that in this region instability waves are indeed present, but do not dominate the velocity fluctuations, especially close to the lipline.

Between $x / D=2$ and the end of the potential core $(x / D \approx 5.5$ for the $M=0.4$ jet $)$ the velocity fluctuations are closely matched by linear PSE, for both axial and radial velocity fluctuarions. However, for locations downstream of the end of the potential core, the agreement between the instability-wave model and the experiment becomes progressively worse, as exemplified in the results for $6 \leq x / D \leq 8$. The comparison of results in this region leads to three hypotheses:

H1. Downstream of the end of the potential core nonlinear effects become significant in the development of wavepackets, invalidating any linear instability-wave model;

H2. Linear instability waves persist for high $x$, but account only for a small part of the azimuthally-coherent overall energy;

H3. The instability-wave Ansatz no longer applies to describe velocity fluctuations in this region: downstream of the potential core the wavepackets degenerate into turbulence.

These hypotheses will be investigated in more detail in the near future, with further exploration of the experimental database. The third possibility seems less likely on account of the persistance of the KelvinHelmholtz shape of disturbance amplitudes, with a zero value close to the lipline for the first POD mode for stations well beyond the potential core, as seen in figure 20. Current work by our group includes nonlinear PSE computations in order to evaluate if inclusion of nonlinear interaction between wavepackets leads to better agreement in the downstream region.

Results representing the development of the instability wave for the helical mode at $\mathrm{St}=0.5$ are shown in figures 22 and 23, for the axial and radial velocity components, respectively. We observe an overall picture 

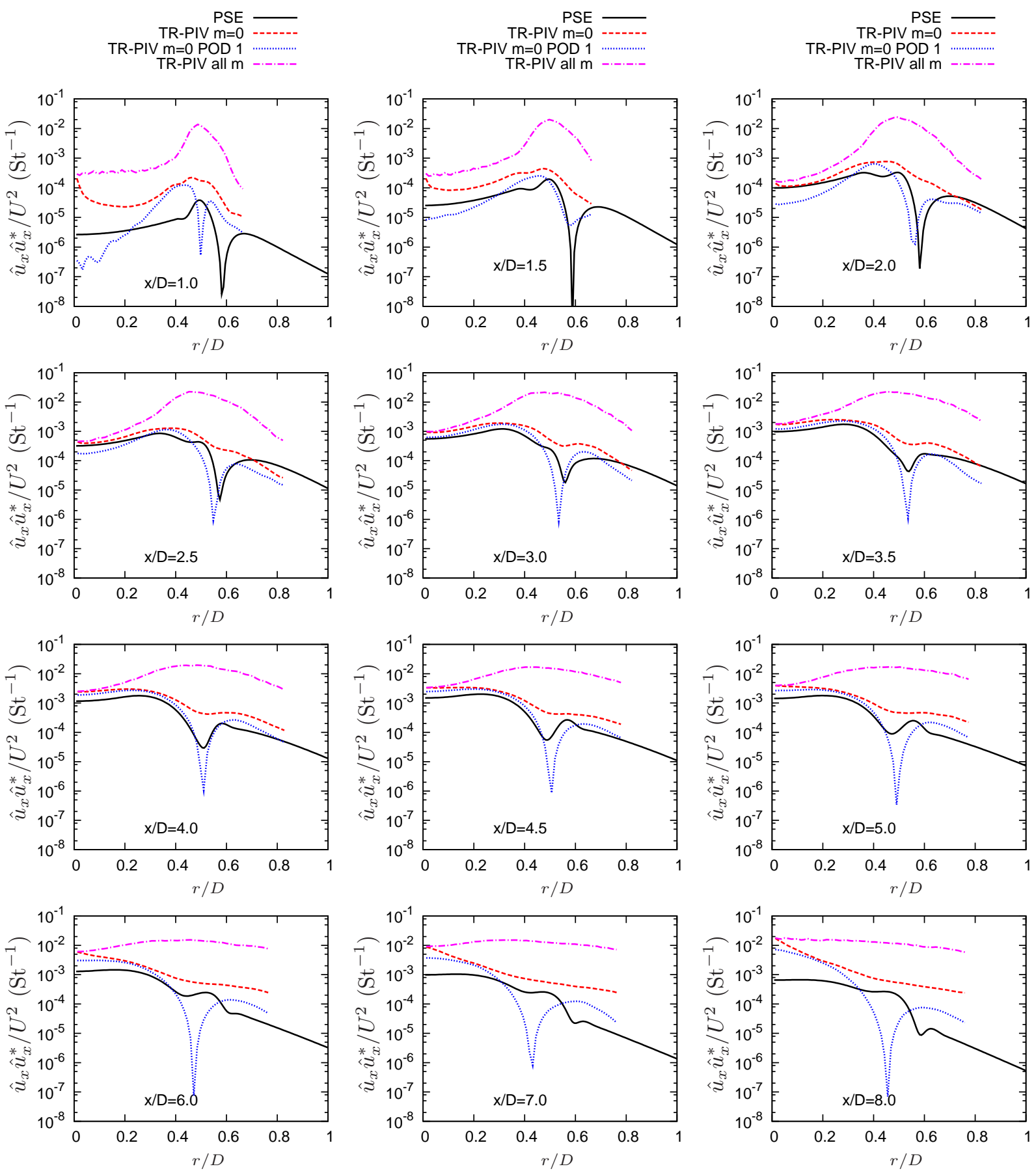

Figure 20. Comparison between $u_{x}$ from linear PSE and experiment for $m=0, M=0.4$ and $\mathrm{St}=0.5 . x / D=1, \mathbf{1 . 5}, \mathbf{2}$, $2.5,3,3.5,4,4.5,5,6,7,8$

similar to the $m=0$ results; however, for $m=1$ the discrepancies between linear PSE and experiment start to become significant for $x / D \approx 4$ for the axial velocity (the radial velocity presents fair agreement up to $x / D=5)$.

To present these results, and also comparisons for other Strouhal numbers, in a more compact manner, we have used the inner product defined in eq. (12) to define a metric $\beta$ of the agreement between linear PSE and experiment, similar to what is done by Gudmundsson and Colonius. ${ }^{16}$ If we define

$$
\beta(x, \omega, m)=\frac{\langle u(x, r, \omega, m), \varphi(x, r, \omega, m)\rangle}{\|u(x, r, \omega, m)\|\|\varphi(x, r, \omega, m)\|}
$$



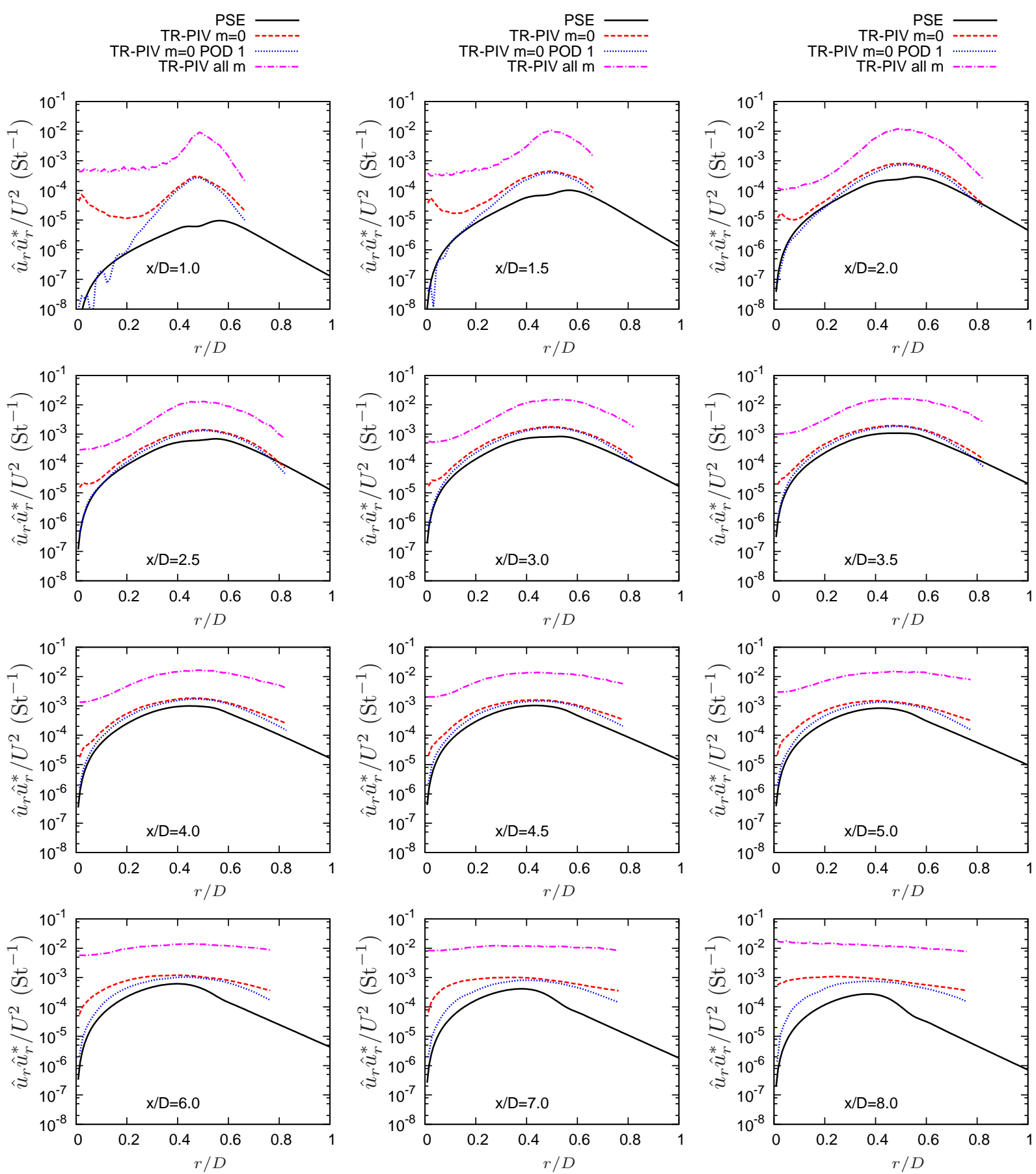

Figure 21. Comparison between $u_{r}$ from linear PSE and experiment for $m=0, M=0.4$ and St $=0.5$. $x / D=1,1.5,2$, $2.5,3,3.5,4,4.5,5,6,7,8$

we have $0 \leq|\beta| \leq 1$, where $|\beta(x, \omega, m)|=1$ means that linear PSE and experiment have exactly the same radial shapes for the three velocity components for given axial station, frequency and azimuthal mode. On the other hand, $|\beta(x, \omega, m)|=0$ indicates orthogonality between model and experiment.

We have calculated the $\beta$ metric using the first POD mode in eq. (15), since it allows the extraction of the coherent part of the instability wave from the experimental results. Results of the metric $|\beta|$ are shown in figures 24 (a) and (b), for azimuthal modes 0 and 1, respectively.

The agreement for the axisymmetric mode is in general better than for the first helical mode. The results in figure 24 show in all cases the three regions aforediscussed. In an initial region, agreement is somewhat 

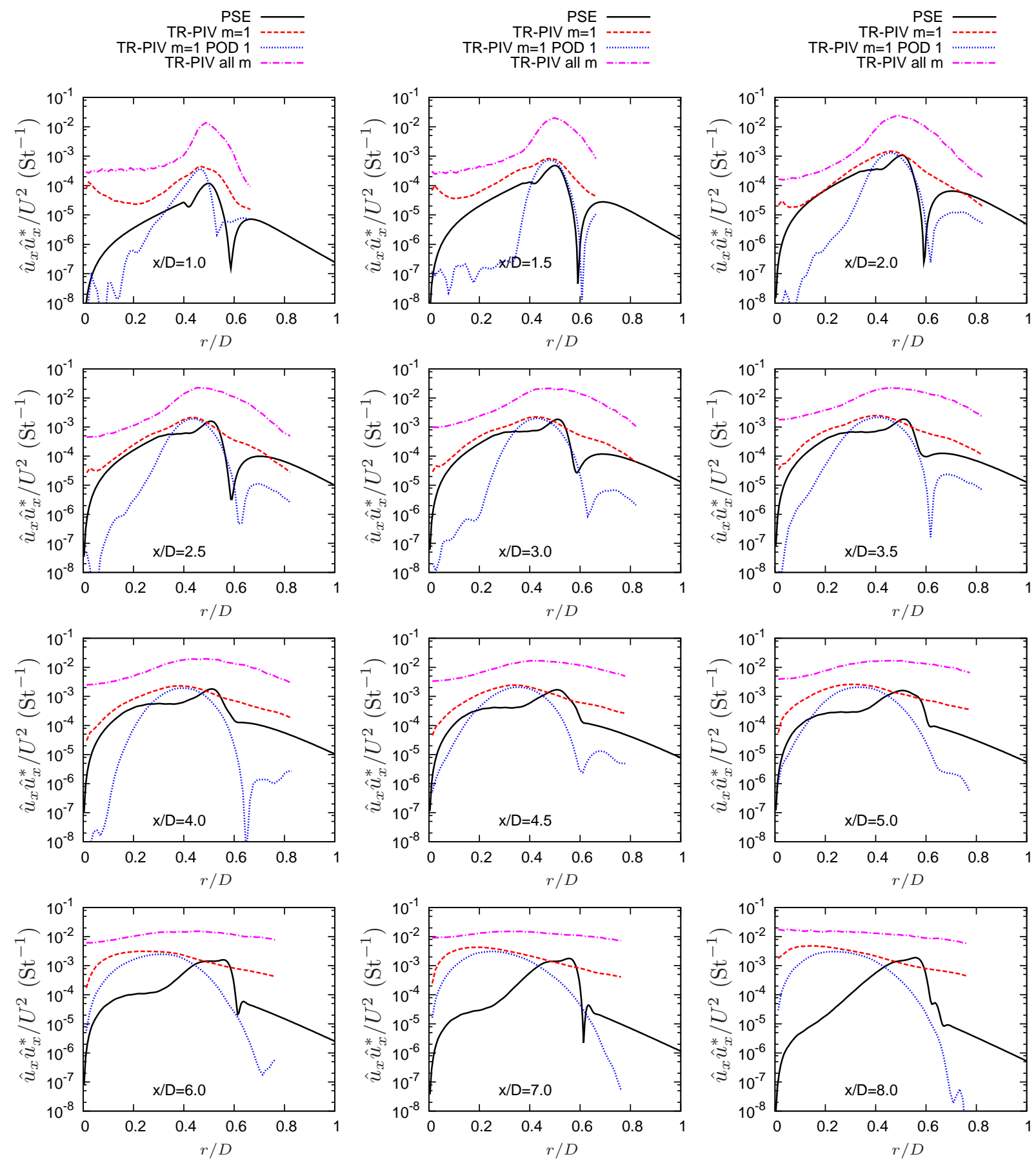

Figure 22. Comparison between $u_{x}$ from linear PSE and experiment for $m=1, M=0.4$ and $\mathrm{St}=0.5 . x / D=1, \mathbf{1 . 5}, \mathbf{2}$, $2.5,3,3.5,4,4.5,5,6,7,8$

worse; this is followed by a region upstream of the end of the potential core with close agreement between PSE and experiment, and at downstream positions discrepancies between model and experiment become significant.

On the other hand, we note that the values of $\beta$ are frequency-dependent. Instability waves with high St tend match better the experimental results for low $x$, and the inverse happens at downstream positions, where agreement with low St is better. This trend is consistent with the growth rates expected for each axial station. For low values of $x / D$, the relatively thinner mixing layer will lead to a high frequency for maximum growth rate, and instability waves with high St will have a fast spatial growth near the nozzle 

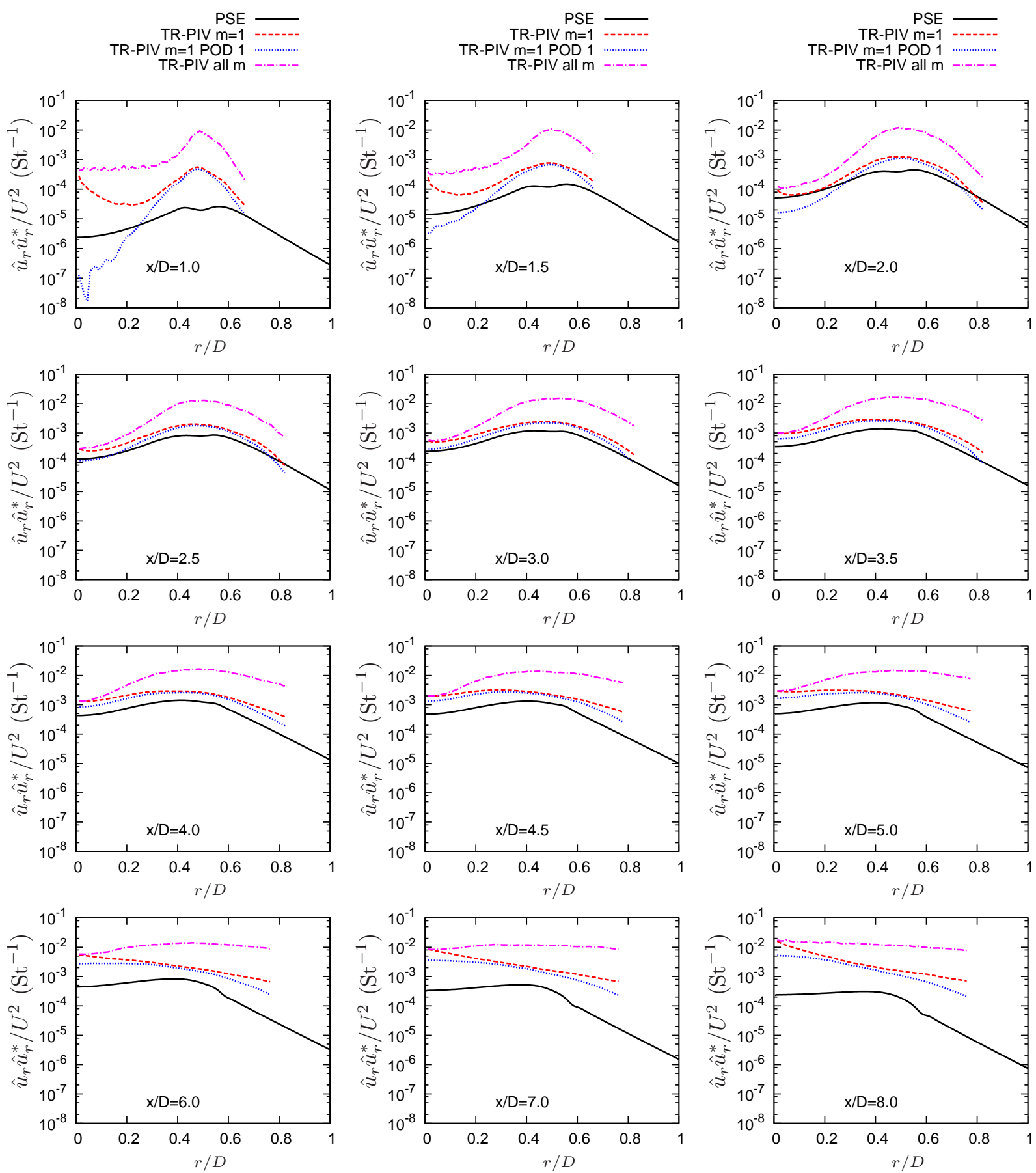

Figure 23. Comparison between $u_{r}$ from linear PSE and experiment for $m=1, M=0.4$ and St $=0.5$. $x / D=1,1.5,2$, $2.5,3,3.5,4,4.5,5,6,7,8$

exit. They tend thus to dominate early the overall fluctuations for a given frequency and azimuthal mode, while low-St waves, with lower growth rate, take a longer extent to have a significant contribution for the measured velocity fluctuations.

This situation is reversed downstream, since the velocity profile becomes progressively stable to high Strouhal numbers, whereas waves with low St are amplified in a longer extent of the jet. The decay of the high-frequency disturbances suggests that the velocity field is no longer dominated, in the downstream region, by instability waves with high Strouhal number. The downstream persistance of low-frequency instability waves may explain their better agreement with the experimental results. 

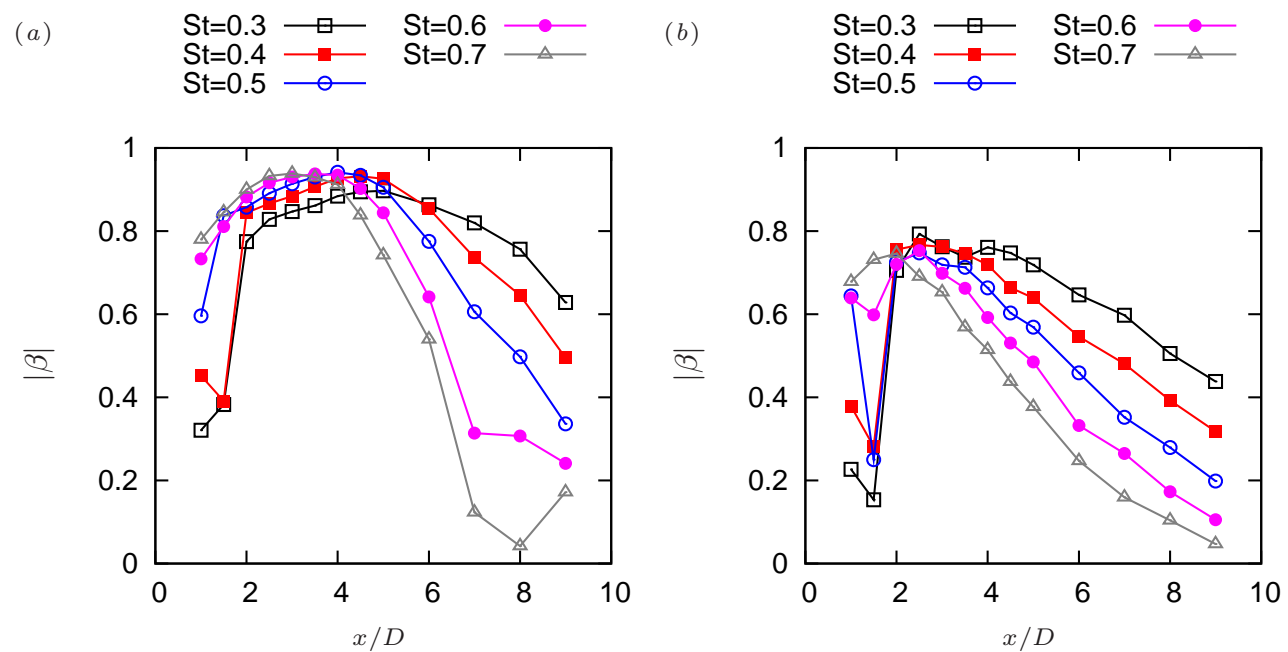

Figure 24. Absolute value of normalised inner product between linear PSE and experiment for (a) $m=0$ and (b) $m=1$.

\section{Conclusion and perspectives}

We present a study of velocity fluctuations in turbulent subsonic jets without externally-imposed forcing, with time-resolved measurements allowing the extraction of azimuthal modes in the flow and in the acoustic field. The velocity field is seen to present significant energy for high azimuthal wavenumbers, whereas lowangle radiation is predominantly axisymmetric. On the other hand, the axisymmetric mode has non-zero amplitude, and when the axisymmetric part of the velocity field is isolated from the turbulence and correlated to the far-field sound, correlations of order of $10 \%$ are obtained, values significantly higher than two-point correlations obtained for these jets and other experiments for subsonic jets.

The velocity field is then studied to verify if this axisymmetric structure, and also the first helical mode, can be described as a superposition of linear instability waves. Linear PSE is used for this, providing a model for linear instability waves accounting for the slow divergence of the experimental mean velocity profiles. There is good agreement for azimuthal modes 0 and 1 until the end of the potential core, showing that linear instability waves are an appropriate model for the development of flucutations in such flows, and that these modes present a wave-packet structure, in agreement with source models identified using far-field information. ${ }^{23,47,48}$

The agreement found between linear PSE and the experimental velocity fluctuations is a further support of the contention that the fluctuations in turbulent jets, for low Strouhal numbers and azimuthal wavenumbers, can be described as linear instability waves with the mean field as a base flow, at least until the end of the potential core. Some nonlinearity is nonetheless implicit, since the base flow in the computations is the experimental mean field. The background turbulence can be seen to establish this mean field via the Reynolds stresses; this base flow now supports a linear Kelvin-Helmholtz instability leading to an extended hydrodynamic wavepacket.

The linearity of the velocity fluctuations contrasts with some numerical studies for low Reynolds number jets, ${ }^{43,49}$ which show that non-linear effects are necessary to describe the evolution of near-field disturbances. In these transitional flows, most of the kinetic energy of fluctuations is related to azimuthally-coherent structures formed during laminar-turbulent transition; these high amplitudes lead to nonlinear effects on the wave-packet evolution, even close to the nozzle exit. However, as seen in section III.A, for a high Reynolds number jet with a turbulent boundary layer in the nozzle, as in the present case, only a small fraction of the turbulent kinetic energy is contained in the low azimuthal modes, which favors application of a linear approach.

The present results show that a part of the turbulent field of high Reynolds number jets can be obtained using the Navier-Stokes equations linearised using the mean velocity field. This is quite different from the general view of turbulence as an essentially nonlinear phenomenon. We doubt though that the dominant turbulent velocity fluctuations, of high azimuthal wavenumber, could be obtained with similar linearisations. On the other hand, if we are looking for the significant velocity fluctuations for sound generation, which have significantly lower energy, a linear wave-packet model appears to be appropriate to describe the evolution 
from the nozzle exit to the end of the potential core, at least in a statistical sense: we note that all comparisons between linear PSE and experiment were done using averaged spectra to obtain amplitudes and phases for each frequency and azimuthal mode. Linear PSE represents thus a good model for a statistical description of large-scale structures.

Downstream of the end of the potential core, though, the model diverges from the experimental results. This can be due, for instance, to significant nonlinear effects in this region, but also to a reduced contribution of the wavepacket to the overall azimuthally-coherent fluctuation energy. Current work involves extension to nonlinear PSE to account for nonlinear interaction of wavepackets, and also a more detailed study of the velocity field to study what happens downstream of the potential core.

\section{Acknowledgments}

We thank Carine Fourment-Cazenave, Patrick Braud and Joël Delville for their work during the experiments. This work was supported by CNPq, National Council of Scientific and Technological Development - Brazil, and through the EU-Russian program ORINOCO (FP7-AAT-2010-RTD-Russia; project number 266103).

\section{Appendix A. Linear PSE comparisons for Mach 0.5 and 0.6}

We show in figures 25 and 26 the comparison between the axisymmetric mode obtained with linear PSE and the experimental results for the axial velocity fluctuations on the jet centerline, respectively, for the $M=0.5$ and 0.6 jets.

The observed trends for both jet Mach numbers are quite similar to the results for the $M=0.4$ jet shown in figure 13. For Strouhal numbers between 0.3 and 0.9 there is close agreement between PSE and the experiment until the end of the potential core. Discussion on the possible reasons for the discrepancies at lower frequencies is presented in section IV.B.

For comparison using the TR-PIV results, increasing the jet Mach number leads to higher aliasing, since the sampling rate was fixed at $5 \mathrm{kHz}$ for all experiments. As a result, the spectral estimates using TR-PIV are significantly higher than the corresponding hot-wire results.

Figures 27 and 28 present comparisons at $x / D=3$ for the axisymmetric mode of the $M=0.6$ jet, for the axial and radial velocity components, respectively. The radial shape of the PSE solution agrees closely with the first POD mode of the experiment. The measured amplitudes are higher than the PSE solution fitted with hot-wire spectra, but this amplitude change seems to be constant with radius.

We have performed an ad hoc correction for aliasing by defining a multiplicative constant for each frequency, which was obtained by dividing the hot-wire spectra on the jet centerline by the TR-PIV spectral estimate for $u_{x}$ at the same position. This same constant was applied to all radial positions, and to both $u_{x}$ and $u_{r}$. Results are shown in figures 29 and 30 . The amplitudes are now quite close, indicating that the shifts in figures 27 and 28 are indeed related to aliasing in the experiments for high Mach number.

\section{Appendix B. Comparison between scalar and vectorial POD}

In this appendix, we compare modes obtained using the scalar POD defined by eq. (9) applied to each velocity component, and a vectorial POD, whose integral equation is given by

$$
\int R_{i, j}\left(x, r, r^{\prime}, m, \omega\right) \xi_{j}\left(x, r^{\prime}, m, \omega\right) r^{\prime} \mathrm{d} r^{\prime}=\lambda(x, m, \omega) \xi_{i}(x, r, m, \omega)
$$

where subscripts $i$ and $j$ refer to the three velocity components $u_{x}, u_{r}$ and $u_{\phi}$, and $R_{i, j}$ is given by

$$
R_{i, j}\left(x, r, r^{\prime}, m, \omega\right)=u_{i}(x, r, m, \omega) u_{j}^{*}\left(x, r^{\prime}, m, \omega\right) .
$$

This vectorial POD is calculated numerically using the approach described by Delville, ${ }^{46}$ with a single $3 N \times 3 N$ matrix formed by computation of $R_{i, j}$ for the three velocity components using the $N$ points in the radial direction. We have used an auxiliar Hermitian kernel, as done by Jung et al. ${ }^{45}$ and described in section IV.C.1.

A sample comparison between results from scalar and vectorial POD is shown in figure 31. For the axial velocity fluctuations, shown in figure 31(a), we note an almost perfect superposition of the first scalar POD 

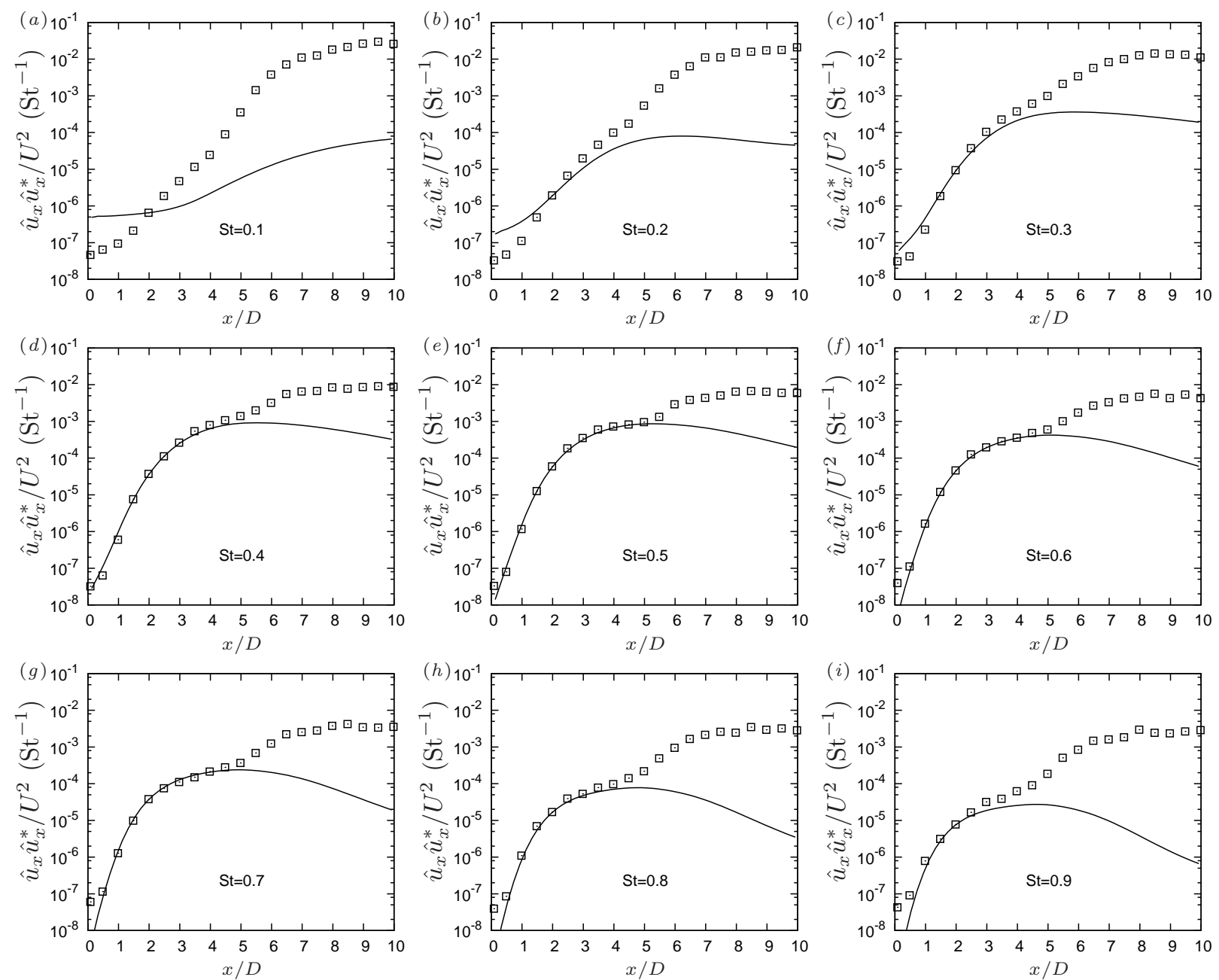

Figure 25. Comparison between linear PSE (lines) for $m=0$ and experimental velocity fluctuations on the centerline (symbols) for the $M=0.5$ jet. Subfigures $(a)-(i)$ refer respectively to Strouhal numbers from 0.1 to 0.9 with increments of 0.1 .

mode and the second vectorial POD mode. For the radial velocity, a similar superposition is found, this time with the first vectorial POD mode.

Since the first scalar POD modes of axial and radial velocity fluctuations were seen in section IV.C to correspond closely to linear stability waves, the results of figure 31 suggest that POD is separating a single physical phenomenon into two different orthogonal modes. This occurs most likely precisely due to the orthogonality requirement, since linear stability modes are known to be non-orthogonal. ${ }^{50}$ In this case, orthogonal modes are not appropriate to describe the considered phenomenon.

Based these observations, we have chosen to use scalar POD for the analysis of the results presented in this paper, the first mode being used mostly as a filter of energetic, radially correlated fluctuations for each velocity component, instead of using vectorial POD.

\section{References}

${ }^{1}$ Crow, S. C. and Champagne, F. H., "Orderly structure in jet turbulence," Journal of Fluid Mechanics, Vol. 48, No. 3, 1971 , pp. $547-591$.

${ }^{2}$ Moore, C. J., "The role of shear-layer instability waves in jet exhaust noise," Journal of Fluid Mechanics, Vol. 80, No. 2, 1977, pp. 321-367.

${ }^{3}$ Michalke, A., "Instabilitat eines Kompressiblen Runden Freistrahls unter Berucksichtigung des Einflusses der Strahlgrenzschichtdicke," Zeitschrift für Flugwissenschaften, Vol. 19, 1971, pp. 319-328; English translation: NASA TM 75190, 1977.

${ }^{4}$ Crow, S. C., "Acoustic gain of a turbulent jet," Phys. Soc. Meeting, Univ. Colorado, Boulder, paper IE, Vol. 6, 1972.

${ }^{5}$ Mankbadi, R. and Liu, J. T. C., "Sound generated aerodynamically revisited: large-scale structures in a turbulent jet as 

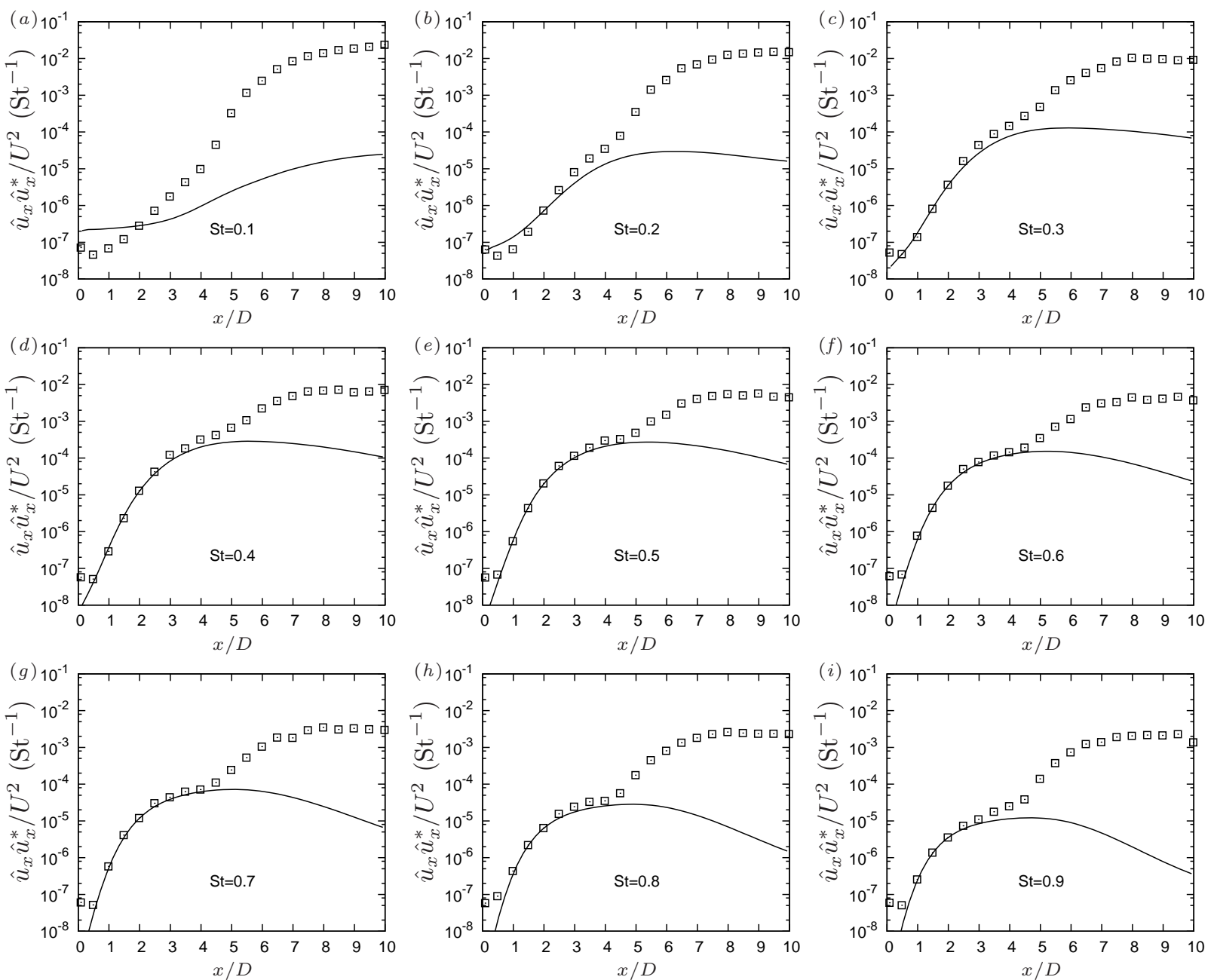

Figure 26. Comparison between linear PSE (lines) for $m=0$ and experimental velocity fluctuations on the centerline (symbols) for the $M=0.6$ jet. Subfigures $(a)-(i)$ refer respectively to Strouhal numbers from 0.1 to 0.9 with increments of 0.1 .
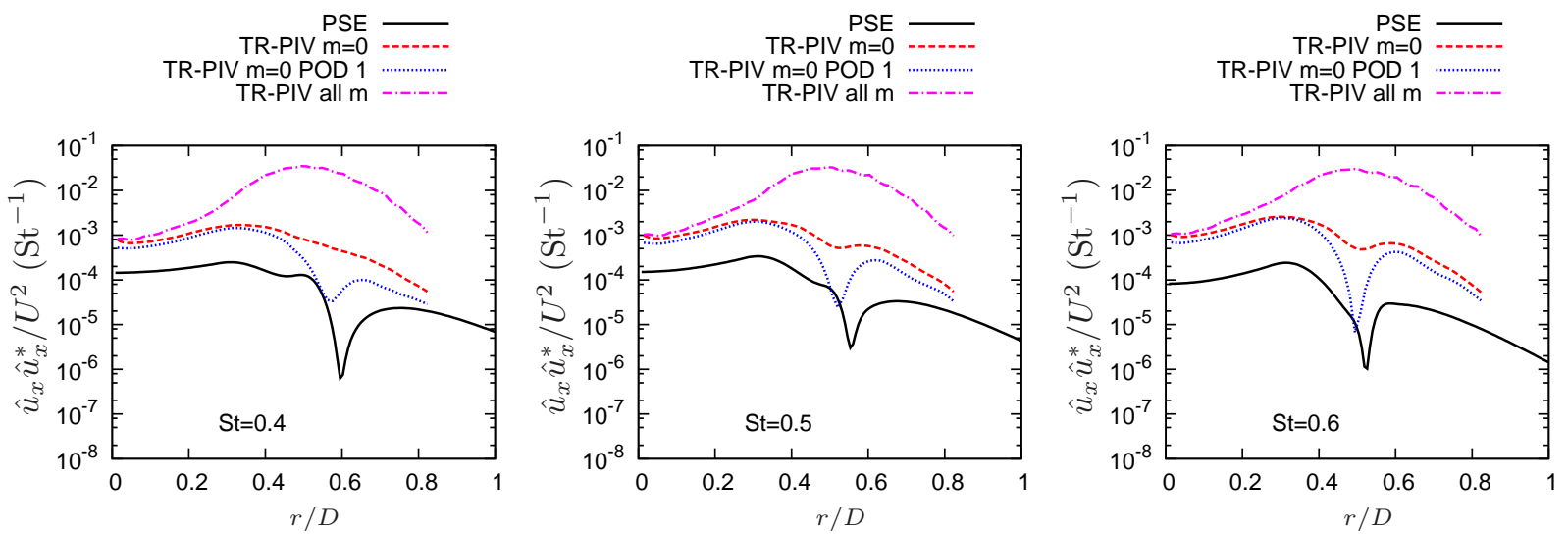

Figure 27. Comparison between $u_{x}$ from linear PSE and experiment for $m=0, M=0.6$ and $x / D=3$

a source of sound," Philosophical Transactions of the Royal Society of London. Series A, Mathematical and Physical Sciences, Vol. 311, No. 1516, 1984, pp. 183-217.

${ }^{6}$ Tam, C. K. W. and Burton, D. E., "Sound generated by instability waves of supersonic flows. Part 2. Axisymmetric jets," Journal of Fluid Mechanics, Vol. 138, No. -1, 1984, pp. 273-295.

${ }^{7}$ Lighthill, M. J., "On sound generated aerodynamically. I. General theory," Proceedings of the Royal Society of London. 

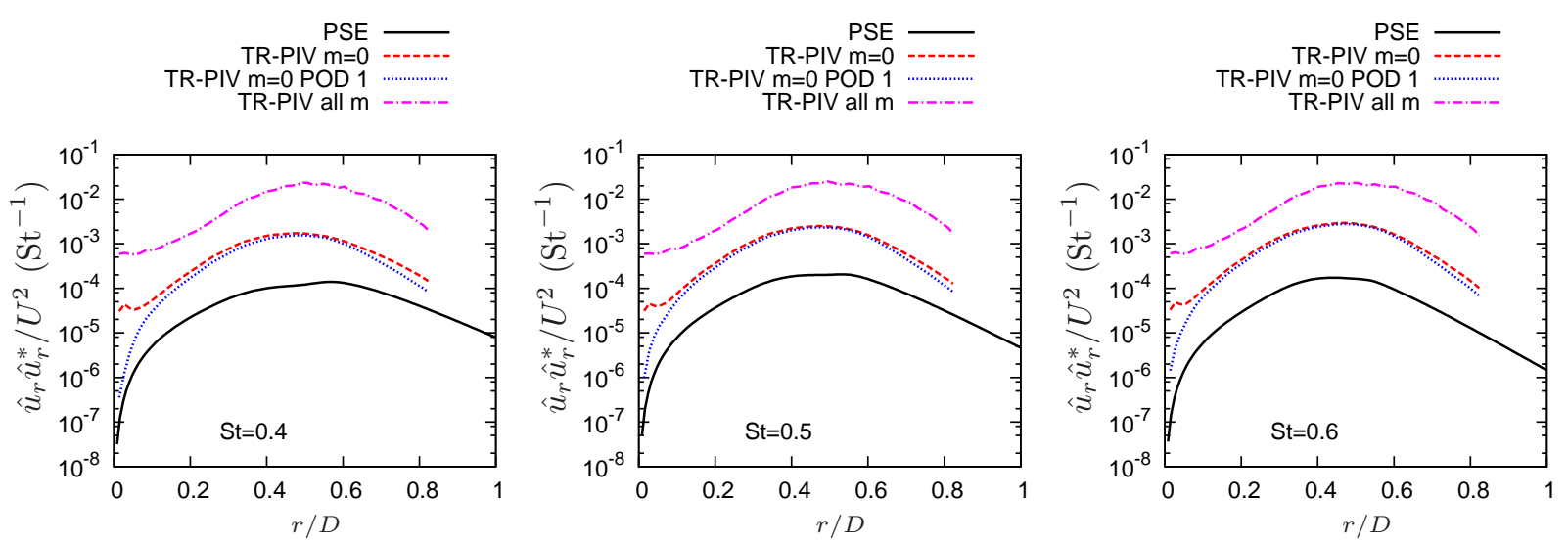

Figure 28. Comparison between $u_{r}$ from linear PSE and experiment for $m=0, M=0.6$ and $x / D=3$
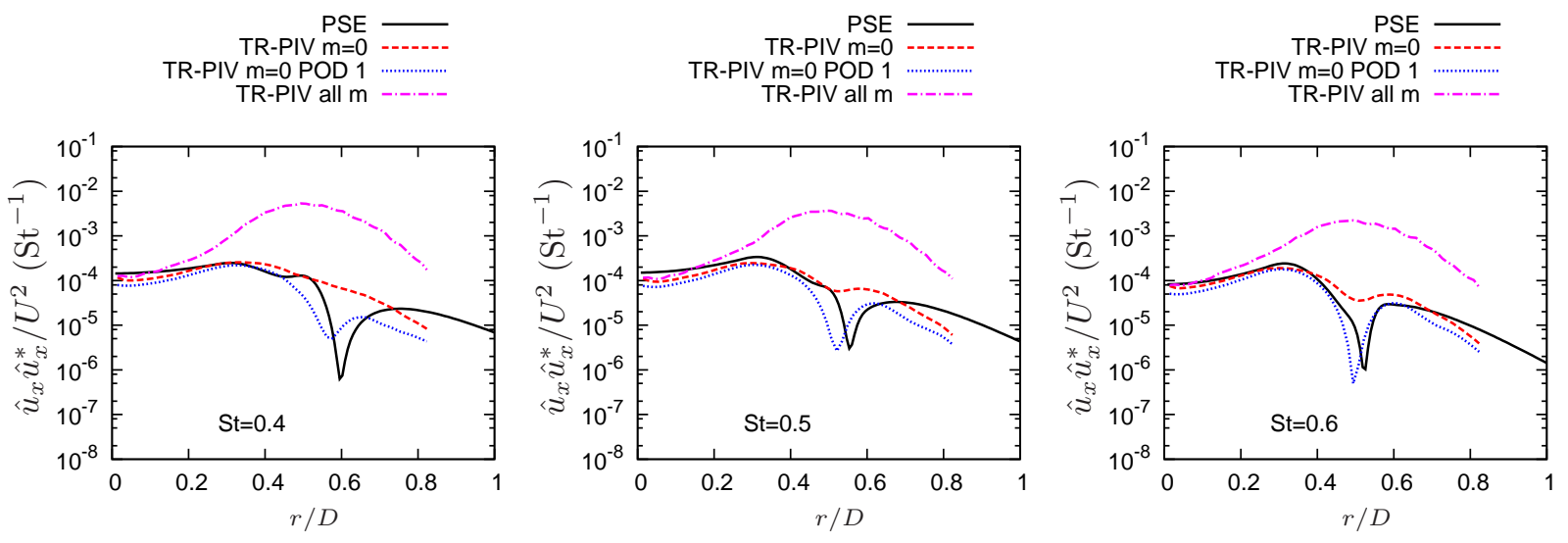

Figure 29. Comparison between $u_{x}$ from linear PSE and experiment (with aliasing correction) for $m=0, M=0.6$ and $x / D=3$
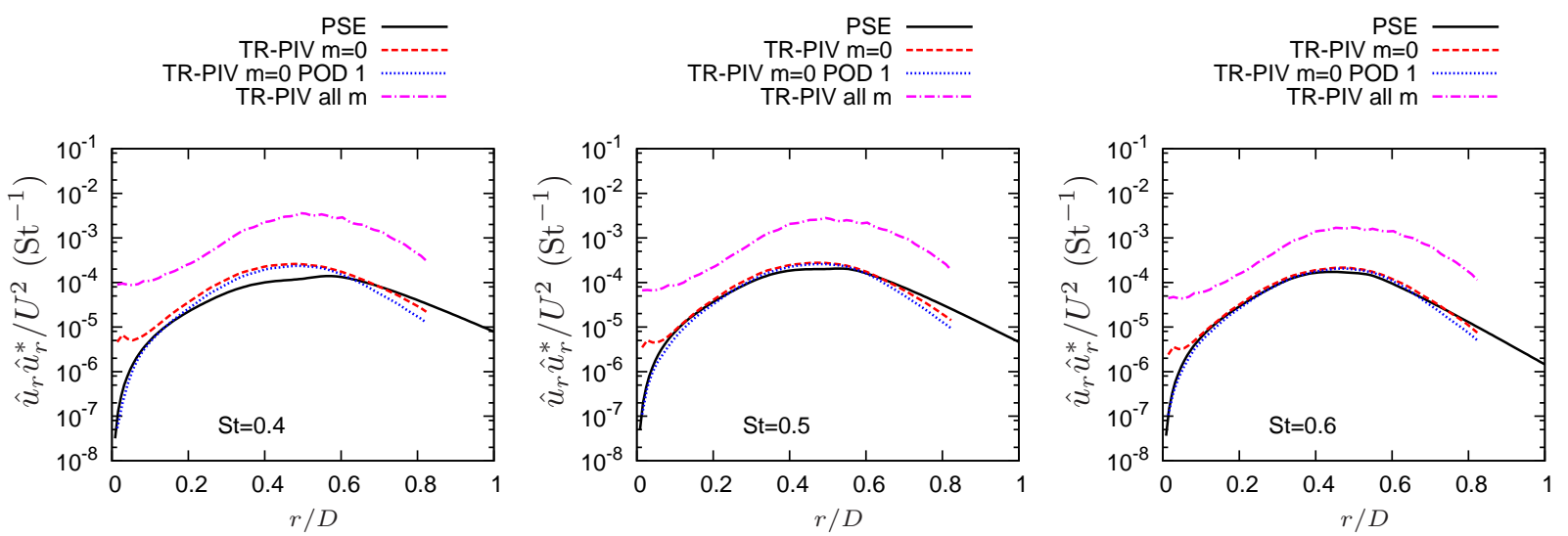

Figure 30. Comparison between $u_{r}$ from linear PSE and experiment (with aliasing correction) for $m=0, M=0.6$ and $x / D=3$

Series A, Mathematical and Physical Sciences, 1952, pp. 564-587.

${ }^{8}$ Cohen, J. and Wygnanski, I., "The evolution of instabilities in the axisymmetric jet. Part 1 . The linear growth of disturbances near the nozzle," Journal of Fluid Mechanics, Vol. 176, 1987, pp. 191-219.

${ }^{9}$ Petersen, R. A. and Samet, M. M., "On the preferred mode of jet instability," Journal of Fluid Mechanics, Vol. 194, No. 1, 1988, pp. 153-173.

${ }^{10}$ Michalke, A. and Fuchs, H. V., "On turbulence and noise of an axisymmetric shear flow," Journal of Fluid Mechanics, Vol. 70, 1975, pp. 179-205.

${ }^{11}$ Armstrong, R. R., Fuchs, H. V., and Michalke, A., "Coherent Structures in Jet Turbulence and Noise," AIAA Journal, 
(a)

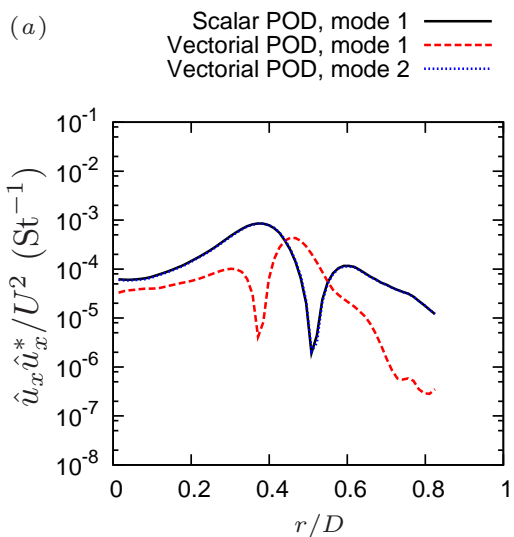

$(b)$

Scalar POD, mode 1 Vectorial POD, mode Vectorial POD, mode 2

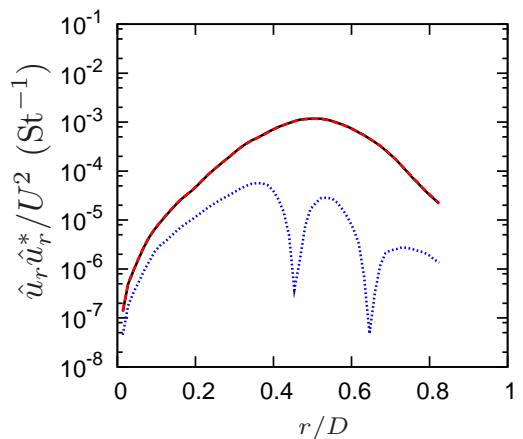

Figure 31. Comparison of scalar and vectorial POD applied to the $M=0.4$ jet for $x / D=2, m=0$, St $=0.6$. Note the superposed curves (first scalar mode is superposed to the second vectorial mode in $a$ and to the first vectorial mode in $b)$.

Vol. 15, 1977, pp. 1011-1017.

${ }^{12}$ Fuchs, H. V. and Michel, U., "Experimental evidence of turbulent source coherence affecting jet noise," AIAA Journal, Vol. 16, No. 9, 1978, pp. 871-872.

${ }^{13}$ Picard, C. and Delville, J., "Pressure velocity coupling in a subsonic round jet," International Journal of Heat and Fluid Flow, Vol. 21, No. 3, 2000, pp. 359-364.

${ }^{14}$ Tinney, C. E. and Jordan, P., "The near pressure field of co-axial subsonic jets," Journal of Fluid Mechanics, Vol. 611, 2008, pp. 175-204.

${ }^{15}$ Suzuki, T. and Colonius, T., "Instability waves in a subsonic round jet detected using a near-field phased microphone array," Journal of Fluid Mechanics, Vol. 565, 2006, pp. 197-226.

${ }^{16}$ Gudmundsson, K. and Colonius, T., "Instability wave models for the near-field fluctuations of turbulent jets," Journal of Fluid Mechanics, Vol. 689, 2011, pp. 97-128.

${ }^{17}$ Kerhervé, F., Jordan, P., Cavalieri, A. V. G., Deville, J., Bogey, C., and Juvé, D., "Educing the source mechanism associated with downstream radiation in subsonic jets," submitted to Journal of Fluid Mechanics, 2012.

${ }^{18}$ Hussain, A. K. M. F. and Zaman, K. B. M. Q., "The 'preferred mode' of the axisymmetric jet," Journal of Fluid Mechanics, Vol. 110, 1981, pp. 39-71.

${ }^{19}$ Becker, H. A. and Massaro, T. A., "Vortex evolution in a round jet," Journal of Fluid Mechanics, Vol. 31, No. 03, 1968, pp. $435-448$

${ }^{20}$ Yule, A., "Large-scale structure in the mixing layer of a round jet," Journal of Fluid Mechanics, Vol. 89, No. 3, 1978, pp. $413-432$

${ }^{21}$ Laufer, J. and Yen, T.-C., "Noise generation by a low-Mach-number jet," Journal of Fluid Mechanics, Vol. 134, 1983, pp. $1-31$.

${ }^{22}$ Violato, D. and Scarano, F., "Three-dimensional evolution of flow structures in transitional circular and chevron jets," Physics of Fluids, Vol. 23, No. 12, 2011, pp. 124104-124104.

${ }^{23}$ Cavalieri, A. V. G., Jordan, P., Colonius, T., and Gervais, Y., "Axisymmetric superdirectivity in subsonic jets," 17 th AIAA/CEAS Aeroacoustics Conference and Exhibit, Portland, OR, USA, June 5-8 2011.

${ }^{24}$ Tutkun, M., George, W. K., Foucaut, J. M., Coudert, S., Stanislas, M., and Delville, J., "In situ calibration of hot wire probes in turbulent flows," Experiments in Fluids, Vol. 46, No. 4, 2009, pp. 617-629.

${ }^{25}$ Konig, M., Fourment-Cazenave, C., Jordan, P., and Gervais, Y., "Jet noise reduction by fluidic injection from a rotating plug," 17th AIAA/CEAS Aeroacoustics Conference and Exhibit, Portland, OR, USA, June 5-8 2011.

${ }^{26}$ Tinney, C. E., Glauser, M. N., and Ukeiley, L. S., "Low-dimensional characteristics of a transonic jet. Part 1. Proper orthogonal decomposition," Journal of Fluid Mechanics, Vol. 612, 2008, pp. 107.

${ }^{27}$ Lee, H. K. and Ribner, H. S., "Direct correlation of noise and flow of a jet," The Journal of the Acoustical Society of America, Vol. 52, 1972, pp. 1280.

${ }^{28}$ Schaffar, M., "Direct measurements of the correlation between axial in-jet velocity fluctuations and far field noise near the axis of a cold jet," Journal of Sound and Vibration, Vol. 64, No. 1, 1979, pp. 73-83.

${ }^{29}$ Juvé, D., Sunyach, M., and Comte-Bellot, G., "Intermittency of the noise emission in subsonic cold jets," Journal of Sound and Vibration, Vol. 71, 1980, pp. 319-332.

${ }^{30}$ Panda, J., Seasholtz, R. G., and Elam, K. A., "Investigation of noise sources in high-speed jets via correlation measurements," Journal of Fluid Mechanics, Vol. 537, 2005, pp. 349-385.

${ }^{31}$ Bogey, C. and Bailly, C., "An analysis of the correlations between the turbulent flow and the sound pressure fields of subsonic jets," Journal of Fluid Mechanics, Vol. 583, 2007, pp. 71-97.

${ }^{32}$ Tomkins, C. D. and Adrian, R. J., "Energetic spanwise modes in the logarithmic layer of a turbulent boundary layer," Journal of Fluid Mechanics, Vol. 545, 2005, pp. 141-162.

${ }^{33}$ Maestrello, L., "Two-point correlations of sound pressure in the far field of a jet: experiment," Tech. rep., National Aeronautics and Space Administration, 1976, NASA TM X-72835. 
${ }^{34}$ Juvé, D., Sunyach, M., and Comte-Bellot, G., "Filtered azimuthal correlations in the acoustic far field of a subsonic jet," AIAA Journal, Vol. 17, 1979, pp. 112. 1970.

${ }^{35}$ Michalke, A., "A wave model for sound generation in circular jets," Tech. rep., Deutsche Luft- und Raumfahrt, November

${ }^{36}$ Cavalieri, A. V. G., Jordan, P., Agarwal, A., and Gervais, Y., "Jittering wave-packet models for subsonic jet noise," Journal of Sound and Vibration, Vol. 330, No. 18-19, 2011, pp. 4474-4492.

${ }^{37}$ Herbert, T., "Parabolized stability equations," Annual Review of Fluid Mechanics, Vol. 29, No. 1, 1997, pp. $245-283$.

${ }^{38}$ Rodríguez, D., Aniruddha, S., Brès, G., and Colonius, T., "Parabolized stability equation models in turbulent supersonic jets," 18th AIAA/CEAS Aeroacoustics Conference and Exhibit, Colorado Springs, CO, USA, June 4-6 2012.

${ }^{39}$ Thompson, K. W., "Time dependent boundary conditions for hyperbolic systems," Journal of Computational Physics, Vol. 68, No. 1, 1987, pp. 1-24.

${ }^{40}$ Mohseni, K. and Colonius, T., "Numerical Treatment of Polar Coordinate Singularities," J. Comp. Phys., Vol. 157, 2000, pp. $787-795$.

${ }^{41}$ Batchelor, G. K. and Gill, A. E., "Analysis of the stability of axisymmetric jets," Journal of Fluid Mechanics, Vol. 14, No. 04 , 1962, pp. 529-551

${ }^{42}$ Sandham, N. D. and Salgado, A. M., "Nonlinear interaction model of subsonic jet noise," Philosophical Transactions of the Royal Society A, Vol. 366, No. 1876, 2008, pp. 2745-2760.

${ }^{43}$ Suponitsky, V., Sandham, N., and Morfey, C., "Linear and nonlinear mechanisms of sound radiation by instability waves in subsonic jets," Journal of Fluid Mechanics, Vol. 658, 2010, pp. 509-538.

${ }^{44}$ Lau, J. C., Fisher, M. J., and Fuchs, H. V., "The intrinsic structure of turbulent jets," Journal of Sound and Vibration, Vol. 22, No. 4, 1972, pp. 379-384.

${ }^{45}$ Jung, D., Gamard, S., and George, W. K., "Downstream evolution of the most energetic modes in a turbulent axisymmetric jet at high Reynolds number. Part 1. The near-field region," Journal of Fluid Mechanics, Vol. 514, 2004, pp. 173-204.

${ }^{46}$ Delville, J., La décomposition orthogonale aux valeurs propres et l'analyse de l'organisation tridimensionnelle des écoulements turbulents cisaillés libres, Ph.D. thesis, Ph. D. thesis, Université de Poitiers, 1995.

${ }^{47}$ Papamoschou, D., "Wavepacket modeling of the jet noise source," 17th AIAA/CEAS Aeroacoustics Conference and Exhibit, Portland, OR, USA, June 5-8 2011.

${ }^{48}$ Konig, M., Cavalieri, A. V. G., Jordan, P., Gervais, Y., and Papamoschou, D., "Far-field filtering for the study of jet noise: Mach and temperature effects," 17th AIAA/CEAS Aeroacoustics Conference and Exhibit, Portland, OR, USA, June 5-8 2011.

${ }^{49}$ Mohseni, K., Colonius, T., and Freund, J. B., "An evaluation of linear instability waves as sources of sound in a supersonic turbulent jet," Physics of Fluids, Vol. 14, 2002, pp. 3593

${ }^{50}$ Schmid, P. J., "Nonmodal stability theory," Annual Review of Fluid Mechanics, Vol. 39, 2007, pp. 129-162. 